\title{
Characterizing turbulent transport in ASDEX Upgrade L-mode plasmas via nonlinear gyrokinetic simulations
}

\author{
D. Told, ${ }^{1}$ F. Jenko, ${ }^{1}$ T. Görler, ${ }^{1}$ F.J. Casson, ${ }^{1}$ E. Fable, ${ }^{1}$ and the ASDEX Upgrade Team ${ }^{1}$ \\ Max Planck Institute for Plasma Physics, EURATOM Association, Boltzmannstr. 2, 85748 Garching, \\ Germany
}

The nature and level of turbulent transport in the outer core of low-confinement (L-mode) discharges performed at the ASDEX Upgrade tokamak [A. Kallenbach et al., Nucl. Fusion 51, 094012 (2011)] are examined. Previously, it was found that for an L-mode discharge of the DIII-D tokamak [J. L. Luxon and L. G. Davis, Fusion Technol. 8, 441 (1985)] gyrokinetic simulations were unable to reproduce the experimental ion heat flux, underestimating it by almost an order of magnitude. In the present work, employing the GENE gyrokinetic turbulence code, an extensive nonlinear study is performed for L-mode discharges of ASDEX Upgrade in order to cross-check this observation. It is shown that no systematic underprediction can be found in these simulations - instead, discrepancies with respect to experimental transport levels are small enough to be resolved within the uncertainties of the experimental profiles. Moreover, it is shown that some turbulence properties resemble closely those of the underlying linear microinstabilities at least out to $90 \%$ of the minor radius, so that quasilinear transport models remain in principle applicable even for these parameters, provided that appropriate nonlinear saturation rules can be developed.

\section{INTRODUCTION}

Characterizing turbulent transport in magnetic confinement fusion devices is one of the key physics challenges on the way to harnessing nuclear fusion for electricity production. ${ }^{1}$ In order to understand and improve the performance of present fusion experiments as well as future reactors, it is necessary to establish a solid understanding of turbulent transport across the whole range of plasma parameters accessible to experiments.

In recent years, in a number of publications ${ }^{2-9}$ the issue of an apparently systematic theoretical underprediction of the experimental heat transport levels in the outer core region of low-confinement (L-mode) tokamak discharges was discussed. This underprediction ('shortfall') was observed in quasilinear gyrofluid predictions using the TGLF model, ${ }^{10,11}$ but as it turned out, also the gyrokinetic turbulence codes GYRO ${ }^{12}$ and GEM $^{13}$ (run at relatively low resolution) failed to recover the experimentally inferred ion heat transport level in a selected case, underpredicting it by a factor of $\sim 7$ (see Ref. 2).

Even though reactor scenarios target H-mode plasmas, for which simulations inside of the edge pedestal do not appear to encounter similar problems, correctly predicting L-mode confinement is important for a number of reasons. In particular, a reliable description of L-mode turbulence is the necessary basis for an understanding of the $\mathrm{L}-\mathrm{H}$ transition, which remains one of the principal open problems of plasma turbulence research. ${ }^{1}$ A more technical implication concerns the accuracy of profile predictions during current-ramp phases, affecting predictions for the Ohmic transformer flux consumption, but also for the requirements placed on the vertical stabilization systems of ITER. ${ }^{14,15}$

The apparent failure of state-of-the-art gyrokinetic simulations to correctly capture L-mode turbulence has drawn much attention in the community, and several groups worldwide have started to investigate such dis- charges with various gyrokinetic codes in order to find an explanation of the observed discrepancies. In addition, a number of ideas about possible elements missing in present theoretical descriptions were put forward. A potential influence of resistive ballooning modes (RBM) was examined, ${ }^{7}$ a suggestion for an extension of existing gyrokinetic simulation codes to higher frequencies was made, ${ }^{16}$ and it was proposed that strongly nonlinear edge turbulence ${ }^{17-19}$ may not have been taken into account properly. Several other possible causes for the discrepancies, including potential numerical deficiencies, nonlocal effects such as avalanches, or turbulence spreading from the edge, were discussed in some of the existing publications. ${ }^{2,6}$

In the present work, we provide a detailed study of turbulent transport in the outer core region of two different L-mode discharges of the ASDEX Upgrade ${ }^{20}$ tokamak, which were designed to exhibit a stationary phase with parameters as usually found in current ramps. It is found that gyrokinetic turbulence simulations are capable of explaining the experimental levels of transport in all the examined cases, without requiring parameter variations outside of the experimental error bars. Carrying out such a study requires a substantial computational effort, however - in the present case, more than 280 separate nonlinear simulations were carried out using the gyrokinetic code GENE, ${ }^{21-23}$ amounting to a computational cost of $\sim 15$ million CPU-hours. Using the gathered nonlinear data, it is shown here that the outer core region of the examined L-mode discharges is in principle accessible to quasilinear modeling, since nonlinear turbulence characteristics such as drift frequencies and phase-shifts between fluctuating quantities remain closely bound to those of the underlying microinstabilities - a property which has been questioned in the discussion of possible reasons for the underprediction observed in the above references. Nevertheless, in order to correctly predict transport levels for the outer region of L-mode plasmas, the 
nonlinear saturation rules employed in present quasilinear transport models need to be generalized.

The paper is structured as follows: In Sec. II, the GENE gyrokinetic code and the methods used for the present study are described, and precise definitions of the employed geometric conventions are given. The results of both nonlinear and linear simulations, along with a detailed physics analysis, are presented in Sec. III. In Sec. IV, we discuss the consequences of the obtained results for quasilinear modeling, and give some suggestions for improvements of the existing models. Finally, a summary of our findings is presented in Sec. V.

\section{METHODOLOGY}

\section{A. Description of gyrokinetic simulation method}

For the present study, numerical simulations were performed using the gyrokinetic turbulence code GENE, retaining at least two particle species (deuterons and electrons) at realistic mass ratio as well as electromagnetic effects. As all of the simulated cases have very low plasma $\beta$, only perpendicular magnetic field fluctuations are retained and the pressure gradient contribution to the curvature drift is neglected. Collisions are treated by means of a linearized Landau-Boltzmann collision operator, which includes both pitch angle and energy scattering as well as modeling terms which ensure conservation of density, momentum, and energy. The flux surface geometry is extracted from SPIDER equilibria ${ }^{4,24}$ (in the form of G-EQDSK files) via a field line tracing method (developed in Refs. 25 and 26, and first applied to ASDEX Upgrade in Ref. 27), through which the metric coefficients of the field-aligned coordinate system are constructed. Effects of equilibrium rotation are neglected throughout the paper, as the analyzed cases exhibit both small rotation velocity $\left(v_{\text {tor }} / v_{\text {th }}\right.$ well below 0.1$)$ and small $E \times B$ shear. In line with these circumstances, momentum transport levels will not be evaluated or compared against the experiment in the present paper.

For the initial simulations, impurities were accounted for only via the effective charge number $Z_{\text {eff }}$, which enters as a prefactor in the collision operator, modifying the collisionality of particles scattering off ions. For the most realistic simulations, though, from which most of the plots in the present paper were generated, boron impurities were included as a separate gyrokinetic species, such that $Z_{\text {eff }}$ matched the experimental value (see Sec. IIID). This assumption represents an upper limit of the experimental boron concentration, and thus a lower limit of the deuterium density, since in reality also other species (e.g., carbon, nitrogen) will contribute to $Z_{\text {eff }}$.

We would like to stress here that numerical settings which suffice for physically accurate simulations of core turbulence will often not do so for parameters of the outer plasma core or the edge, mainly due to the drastically different values of safety factor, magnetic shear, and back- ground profile gradients. Turbulent spectra react to these parameters, thus requiring different box setups and careful verification (see Sec. IIID). In the present article, all resolutions in the plane perpendicular to the background field (i.e. in the directions labeled $x$ and $y$ ) will be given in terms of complex Fourier modes. Due to the hermiticity of the Fourier coefficients for real quantities, a given number of modes $\left(n_{k x}, n_{k y}\right)$ corresponds to twice that number $\left(n_{k x,}, 2 n_{k y}\right)$ of grid points in the $(x, y)$ direction. For the present nonlinear simulations, a typical choice of resolution was $512 \times 64 \times 32 \times 32 \times 16$ points in the radial $(x)$, binormal $(y)$, parallel $(z)$, parallel velocity $\left(v_{\|}\right)$, and magnetic moment $(\mu)$ grids, respectively. Due to the strong magnetic shear $(\hat{s}>2$, see definition in Eq. (2)), a large radial resolution is typically required. The convergence of the results at the chosen resolution was compared against a number of simulations using the shifted-metric approach. ${ }^{28,29}$

The minimum finite toroidal mode number retained in the simulations was usually $2-8$, with convergence checks done to ensure the sufficiency of this choice. Radial box sizes were usually chosen to be approximately $110 \rho_{s}$ (in GENE coordinates, see Sec. IIB). The extent of the simulation domain along the magnetic field corresponds to one poloidal turn, and the velocity grids were set to encompass a region up to $v_{\max }=3 v_{\mathrm{th}, j}$, with the thermal velocity $v_{\mathrm{th}, j}=\sqrt{2 T_{0 j} / m_{j}}$ of the $j$ th species.

The simulations presented here make use of the local approximation, i.e., the relevant turbulent dynamics is assumed to occur on much smaller scales than the variation of background profiles and gradients, enabling the use of periodic radial boundary conditions. A pseudospectral approach is used to solve the resulting system of equations. There are some notable exceptions, though, where we find it necessary to limit the radial extent that turbulent structures can develop. These cases, as well as the methods used to achieve this size limiting, will be described in the text (see Sections III C and IIIF).

\section{B. Geometric definitions}

In order to provide the simulation setups and results given in the present paper in a form that is easily comparable to those of other gyrokinetic codes, we detail the geometric definitions used in the simulations of the present study. In existing benchmark publications (see, e.g., Refs. 30-34), circular geometry was often employed, ${ }^{35,36}$ in which there is practically no ambiguity in the definition of the magnetic coordinates. Even when a local Miller equilibrium was used for benchmarking, ${ }^{37,38}$ the coordinate choices made in the involved codes were often identical. When using realistic geometry, however, differing coordinate setups affect both the meaning of box sizes as well as the definitions of wavenumbers against which spectra are plotted, thus often leading to confusion when comparing output from different codes. To clarify this point, the definitions employed in the GENE interface 
to G-EQDSK files, which serve as the geometry input in this work, are outlined in the following.

The magnetic field in a tokamak can be represented as

$$
\boldsymbol{B}=\nabla \psi \times \nabla(q \chi-\varphi),
$$

where $\psi$ is the poloidal flux divided by $2 \pi, \varphi$ is the toroidal angle, and $\chi$ is the straight field line angle. Thus we can write the magnetic field as $\boldsymbol{B}=\mathcal{C} \nabla x \times \nabla y$, where $x$ is an (in principle) arbitrary radial coordinate, $y=C_{y}(q \chi-\varphi)$ is the binormal coordinate, and $\mathcal{C}=\left(C_{y} \partial_{x} \psi\right)^{-1}$ is a flux-surface dependent prefactor. With the choice

$$
x=\sqrt{\frac{\Phi}{\pi B_{\text {ref }}}}=\sqrt{\frac{\Phi}{\Phi_{\text {edge }}}} \sqrt{\frac{\Phi_{\text {edge }}}{\pi B_{\text {ref }}}}=\rho_{\text {tor }} L_{\text {ref }},
$$

where $\Phi$ is the toroidal flux divided by $2 \pi, B_{\text {ref }}$ is the reference toroidal field taken at the magnetic axis, and $L_{\text {ref }}$ is defined to be a macroscopic reference scale, we can set $C_{y}=\rho_{\text {tor }} L_{\text {ref }} / q$, so that in turn $\mathcal{C}=B_{\text {ref }}$. For global simulations, we choose to define $C_{y}$ as a constant over the entire domain (with $\rho_{\text {tor }}$ and $q$ taken at the radial center of the simulation domain), so that $\mathcal{C}$ in turn picks up a radial variation.

The fact that $C_{y}$ appears as a coefficient in the definition of the $y$ coordinate means that it will affect the definitions of wavenumbers $k_{y}$ in this dimension, complicating direct comparisons between implementations with different definitions. To facilitate benchmarks, it is therefore preferable to convert all output either to a common definition, or to a uniquely defined quantity such as the toroidal mode number. The conversion from GENE $k_{y}$ wavenumbers to the latter is given by

$$
n=\hat{k}_{y} \hat{C}_{y} \rho_{*}^{-1},
$$

where the hat symbol indicates normalized quantities, and the $\rho_{*}=\rho_{\text {ref }} / L_{\text {ref }}$ factor accounts for the different normalizations of $\hat{k}_{y}=k_{y} \rho_{\text {ref }}$ and $\hat{C}_{y}=C_{y} / L_{\text {ref }}$. In GENE, usually $\rho_{\text {ref }}=\rho_{s}=\sqrt{T_{e} m_{i}} / e B_{\text {ref }}$, and $L_{\text {ref }}$ is defined as in Eq. (1) and therefore resembles a fluxsurface averaged minor radius. With these definitions, the box size in binormal $(y)$ direction is then determined by the spacing of toroidal mode numbers $\Delta n$. In Table I, the conversion from $k_{y}$ wavenumbers to toroidal mode number in each of the studied cases is given.

In order to compare radial box sizes, in the case of fluxtube simulations it is convenient to exploit the quantization of the radial box size, which must be enforced due to the periodic radial boundary condition ${ }^{39}$ and restricts the box sizes $L_{x}$ and $L_{y}$ such that

$$
\mathcal{N}=2 \pi \hat{s} \frac{L_{x}}{L_{y}}\left(C_{y} \frac{q_{0}}{x_{0}}\right) \quad \text { with } \hat{s}=\frac{x_{0}}{q_{0}} \frac{d q}{d x}
$$

is an integer number. Here, $x_{0}$ and $q_{0}$ are the radial coordinate and the safety factor of the flux surface containing the simulated flux tube. For $\mathcal{N}=1$ in such a setup,
$L_{x}$ corresponds precisely to the distance between two mode rational surfaces for the $k_{y, \min }=2 \pi / L_{y}$ wavenumber, and the value of $\mathcal{N}$ in a simulation can serve as a coordinate-free measure of the radial box size for given magnetic shear $\hat{s}$. Using the values $\Delta n$ and $\mathcal{N}$, it is therefore possible to specify uniquely the physical box size for a given parameter set. In Table II, the perpendicular extent of the simulation domain is given using the above defined quantities. In the case of non-periodic (but local) simulations, the radial box size is not quantized. The above parameter $\mathcal{N}$ can then still be used as a coordinate-free measure, but it is no longer restricted to integer numbers. For global simulations, the toroidal mode number spacing is still a useful quantity for comparison, but radial domain sizes are best compared in a flux label coordinate (here: $\rho_{\text {tor }}$ ).

Taking into account these conventions, linear and nonlinear benchmarks between GENE and the gyrokinetic GKW code $^{40}$ were carried out for the ASDEX Upgrade discharges studied in this work. These simulations, although performed at somewhat lower resolution than the ones shown in Sec. III C, retained both numerical magnetic geometry and collisions and found very good agreement between both codes. The linear results of this comparison, as well as the transport levels obtained from nonlinear simulations, were shown already in Ref. 41. In addition to these results, a more stringent comparison of the nonlinear spectra obtained from both codes is shown in App. A.

\section{GYROKINETIC SIMULATIONS}

\section{A. Physical parameters}

In the framework of the present study, we examine four different operation points, whose physical parameters are taken from the discharges 28132 and 28151 of the ASDEX Upgrade tokamak. Both of these discharges had a toroidal magnetic field of $2.3 \mathrm{~T}$, but differing plasma currents of $400 \mathrm{kA}\left(q_{95} \approx 12\right)$ in discharge 28132 , and 800 $\mathrm{kA}\left(q_{95} \approx 6\right)$ in discharge 28151 . In both discharges, a small amount of electron cyclotron heating was applied, compensating approximately for the difference in Ohmic heating.

For the high-power phase of each discharge (28132: $\left.P_{\mathrm{ECRH}}=1.16 \mathrm{MW}, 28151: P_{\mathrm{ECRH}}=0.53 \mathrm{MW}\right)$, local GENE simulations are performed at the positions $r / a=0.75$ and $r / a=0.85$, where $r$ is defined to be the local minor radius of a surface (given as $\left(R_{\text {out }}-R_{\text {in }}\right) / 2$ ), measured at the average elevation of the flux surface - as defined also in Ref. 42, and $a$ is the minor radius of the last closed flux surface. The four main operation points that will be analyzed in the present paper will be labeled with the letters A through D. The physical parameters for each of these four cases are given in Table I. There, 
the logarithmic gradients are defined as

$$
\omega_{X}=-\frac{1}{X} \frac{d X}{d \rho_{\mathrm{tor}}}
$$

with $X \in\left\{T_{i}, T_{e}, n_{e}\right\}$, the magnetic shear is given as

$$
\hat{s}=\frac{\rho_{\mathrm{tor}}}{q} \frac{d q}{d \rho_{\mathrm{tor}}},
$$

the electron beta is defined as $\beta_{e}=2 \mu_{0} n_{e} T_{e} / B_{\text {ref }}^{2}$, and the electron-ion collisionality is given as

$$
\nu_{e i}=\frac{Z_{\mathrm{eff}} n_{e} e^{4} \ln \Lambda}{2^{7 / 2} \pi \epsilon_{0}^{2} m_{e}^{1 / 2} T_{e}^{3 / 2}} .
$$

In addition to the operation points examined in the present work, the plasma parameters of the DIII-D ${ }^{43}$ "shortfall" case $^{2}$ are shown for comparison. As can be seen in Table I, our plasma parameters are generally quite similar to that case, except for the ion/electron temperature ratio (due to NBI vs. ECRH heating) and the $Z_{\text {eff }}$ value (due to carbon vs. tungsten wall materials). We therefore expect the presently studied cases to be highly relevant for further investigation of the shortfall issue. In a forthcoming publication, an extensive study of the DIII-D shortfall case itself, using the GENE code, will be detailed.

\section{B. Microinstability investigations}

In order to establish a physical picture of the relative strength of the drive mechanisms of the turbulence, it is useful to study the linearized system, since certain characteristics of linear physics often persist in the fully developed nonlinear turbulent state. ${ }^{22,44-48}$ In the present case it is particularly interesting to follow this approach, since it must be clarified whether quasilinear transport modeling is applicable to the outer region of L-mode plasmas.

For each of the four operation points, we scan the full range of binormal wavenumbers from machine size scales down to electron gyroradius scales; in addition to the common ion temperature gradient (ITG) or trapped electron mode instabilities (TEM), we therefore also retain microtearing modes (MTM) and electron temperature gradient (ETG) instabilities in the linear analysis. Furthermore, subdominant instabilities are tracked employing the eigenvalue solver integrated into GENE, ${ }^{49-51}$ which allows a more complete comparison between linear and nonlinear transport characteristics. For the present linear simulations, we employ a resolution of $31 \times 64 \times 64 \times 16$ in $\left(k_{x}, z, v_{\|}, \mu\right)$ dimensions, analyzing a single $k_{y}$ mode at a time.

In Figs. 1 and 2, growth rates and mode frequencies for all four operation points are shown, retaining two kinetic species (deuterium ions and electrons). In all figures, black lines and symbols belong to the dominant instability, while red lines and markers indicate the strongest
Table I: Physical parameters for the four simulated ASDEX Upgrade L-mode cases, in comparison to the DIII-D shortfall case. ${ }^{2}$ The reference length is defined as $L_{\text {ref }}=\sqrt{\Phi_{\text {edge }} / \pi B_{\text {ref }}}$.

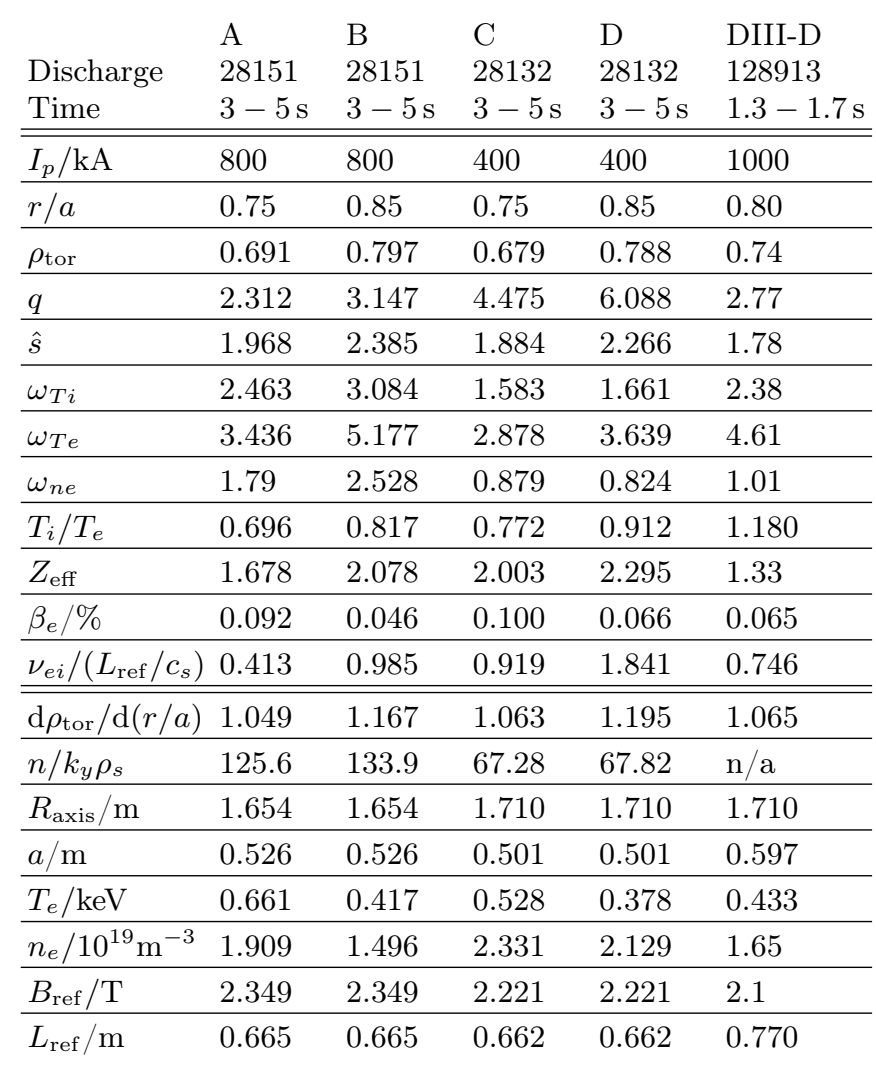

subdominant instability, if present. Electron and ion drift directions are designated by crosses and diamonds, respectively.

An analysis of the simulation results reveals that all of the instabilities mentioned above are found in the present cases, in addition to a hybrid instability with mixed drive. We start by describing the results for cases C and D (the low-current discharge at 0.4 MA), since the behavior of these two cases is very similar, and straightforwardly explained. Going from low to high wavenumbers, both cases are dominated first by a microtearing mode up to $k_{y} \rho_{s} \sim 0.1$, then by an ITG mode up to $k_{y} \rho_{s} \sim 0.7 / 0.3$ (case C/D), and a combined TEM-ETG branch at higher wavenumbers. Furthermore, in the low- $k_{y}$ regime up to $k_{y} \rho_{s}=2$, the most unstable subdominant mode is always one of the instabilities just described - there is first a coexistence of MTM and ITG, and at higher $k_{y} \rho_{s}$ a coexistence of ITG and TEM. Only for $k_{y} \rho_{s} \gtrsim 10$, an additional subdominant eigenmode of ETG type is found. The main difference between the linear behavior of cases $\mathrm{C}$ and $\mathrm{D}$ is the earlier, and stronger, onset of the trapped-electron mode at $k_{y} \rho_{s} \sim 0.3$ in case $\mathrm{D}$, hinting at an increased fraction of electron heat transport at that radial position. As will be discussed in Sec. III C, the microtearing activity found at low wavenumbers in both cases can lead 

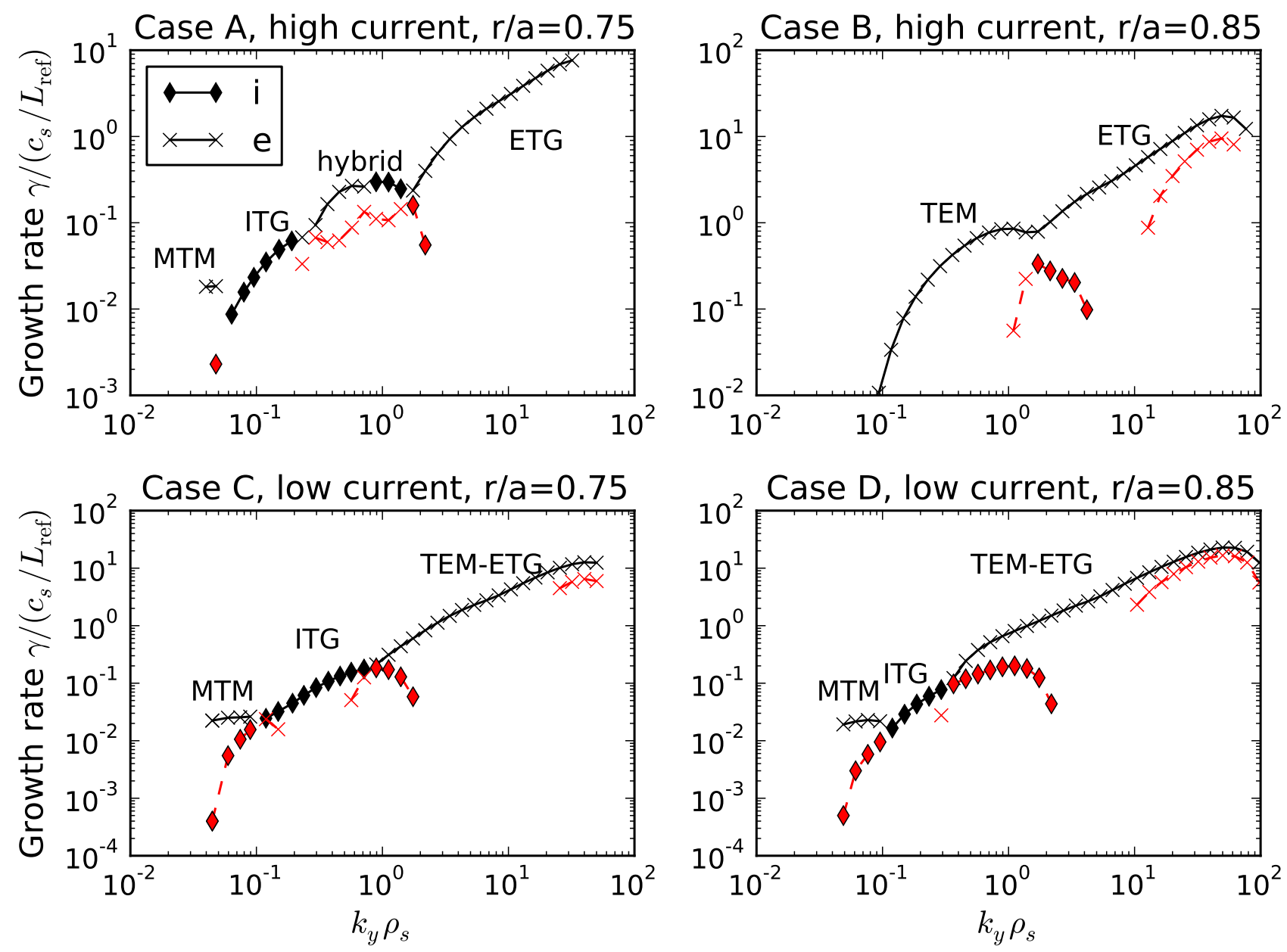

Figure 1: (Color online) Linear growth rates for the four operation points A-D. The labels 'i' and 'e' refer to modes drifting in the ion or electron diamagnetic direction, respectively, and black solid (red dashed) color marks the dominant (subdominant) instability. The labels in the plots refer to the most unstable mode. See main text for further physical interpretation, and Tab. I for the definitions of $L_{\mathrm{ref}}$.

to numerical problems in nonlinear simulations, related to the large radial extent of these instabilities.

For the high-current cases $\mathrm{A}$ and $\mathrm{B}$, the linear behavior is somewhat less transparent: For the $r / a=0.75$ position, the situation is similar to the cases described above. At the lowest wavenumbers, an unstable microtearing instability is found, which is then followed by an ITG instability at higher wavenumbers between 0.05 and 0.2 . Beyond these wavenumbers, however, a hybrid mode takes over (rather than a 'pure' TEM as in cases C and D), whose mode frequency exhibits a smooth sign change from electron to ion drift direction around $k_{y} \rho_{s} \sim 0.8$. Another hybrid mode, but with lower growth rate, appears above $k_{y} \rho_{s} \sim 0.3$ and connects to the ETG branch, which dominates beyond $k_{y} \rho_{s} \sim 2$. In case $\mathrm{B}$, on the other hand, no unstable microtearing mode is found. Instead, a trapped electron mode governs the entire range from $k_{y} \rho_{s}=0.1$ up to $k_{y} \rho_{s} \sim 2$, beyond which an ETG instability is the most unstable mode. In the low- $k_{y}$ range up to $k_{y} \rho_{s} \sim 1$, no subdominant instabilities are found here.

Note that ETG modes are unstable in all four cases, but their relative growth rates (compared to the instabilities at larger scales) are stronger in the $0.4 \mathrm{MA}$ discharge, where they exceed their low- $k$ counterparts by roughly a factor of 50, indicating possibly important transport contributions from small-scale ETG turbulence. ${ }^{52}$ In that discharge, there are also more pronounced microtearing instabilities, hinting at the possible importance of electromagnetic effects. As will be discussed in Sec. III C, this microtearing activity is indeed reflected also in the turbulent state and poses a practical challenge due to its numerical implications (see also Refs. 53-55 for simulations of pure microtearing turbulence).

In summary, linear simulations show that most of the examined operation points exhibit a coexistence of TEM- 

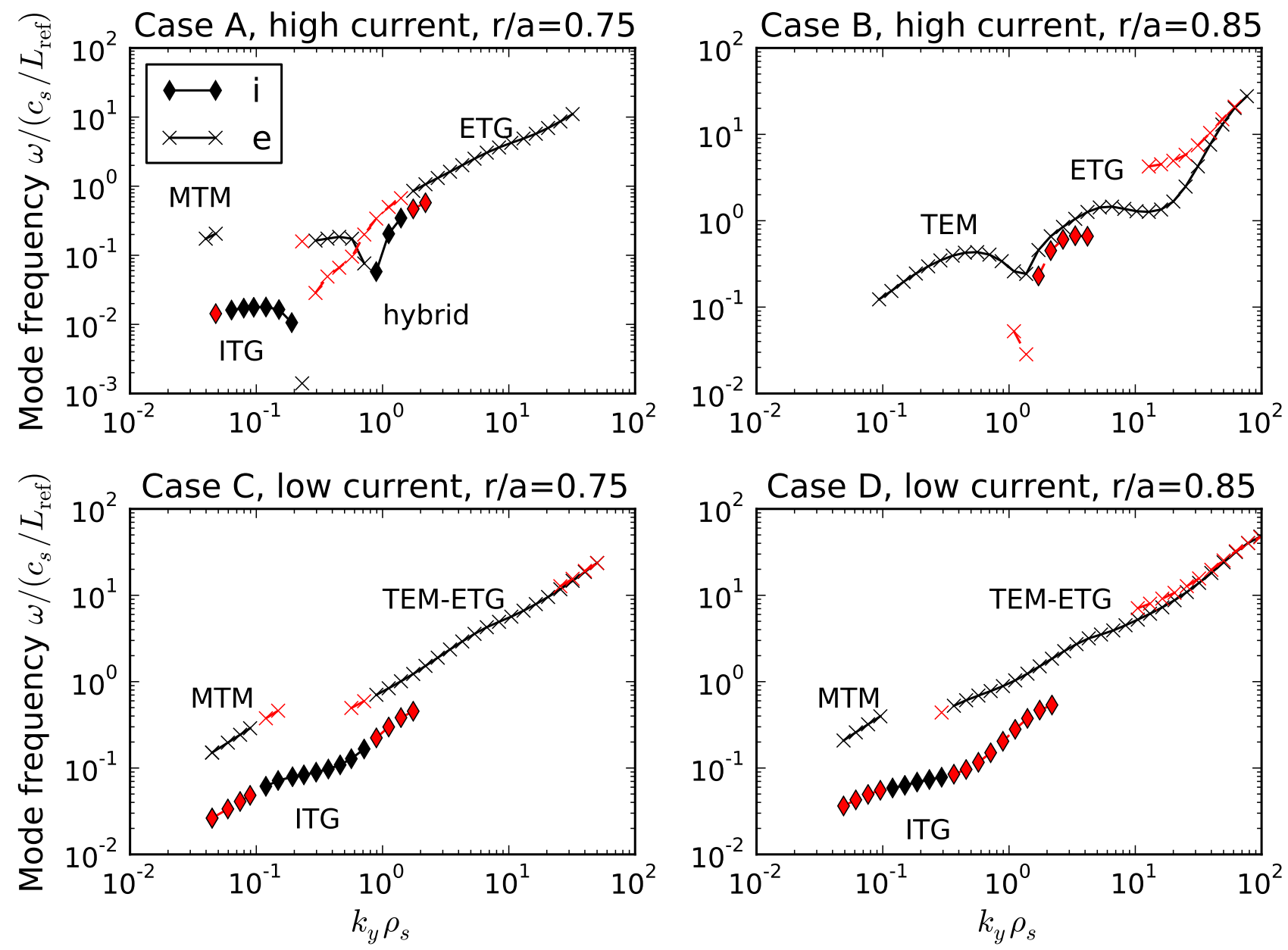

Figure 2: (Color online) Linear mode frequencies for the four operation points A-D. As in Fig. 1, the labels 'i' and 'e' refer to modes drifting in the ion or electron diamagnetic direction, respectively, and black solid (red dashed) color marks the dominant (subdominant) instability. The labels in the plots refer to the most unstable mode. See main text for further physical interpretation, and Tab. I for the definitions of $L_{\text {ref }}$.

type and ITG-type instabilities, except for case B in which ITG modes at low $k_{y}$ are stable. At the lowest examined wavenumbers, microtearing modes are found, primarily for the parameters of the low current (high safety factor) discharge. While the nonlinear simulations discussed in the following sections will focus on these large-scale instabilities, all four cases also exhibit unstable ETG modes, whose contribution to turbulent transport will be studied in Sec. IIIF.

\section{Turbulence simulations}

\section{Setup}

In this section, the nonlinear, turbulent regime for each of the four operation points A-D is explored. The physical parameters are those listed in Table I; the numerical parameters are shown in Table II, with the most realistic simulations (from which all of the following figures were produced) printed in boldface.

All simulations are set up such that most of the dissipation is caused by collisions and therefore physical. Numerical dissipation is introduced in the parallel direction in order to damp grid-scale modes, and in some cases also in the perpendicular plane, if spectral pile-up (e.g., due to unresolved ETG contributions) is found in the simulations without perpendicular dissipation. In the present work, all the numerical dissipation terms have the form of fourth-order hyperdiffusion terms (see Ref. 56). Apart from these supplementary dissipative terms, all Poisson bracket terms that appear in the gyrokinetic equation have been discretized such that their analytical conservation properties are being retained in the numerical representation ${ }^{57}$-namely, an Arakawa ${ }^{58}$ discretization is employed for the parallel dynamics, whereas the conser- 


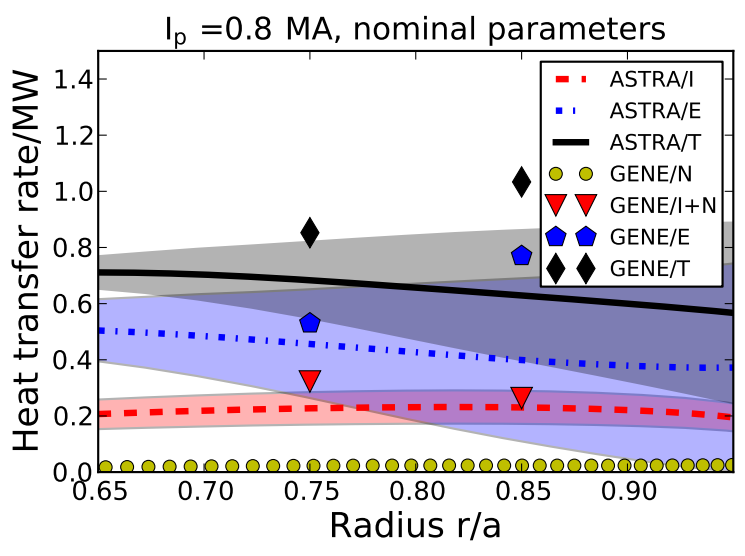

(a)

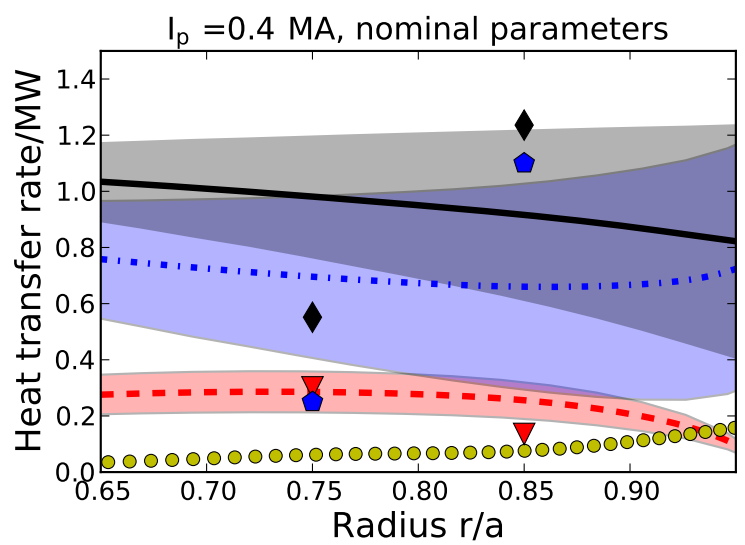

(b)

Figure 3: (Color online) Comparison of experimental fluxes (lines) with those obtained from ion-scale GENE simulations (markers), using nominal parameters (but including boron impurities) for the a) $0.8 \mathrm{MA}, \mathrm{b}) 0.4 \mathrm{MA}$ discharge (simulations are marked in boldface in Table II). The total heat transfer rate obtained from ASTRA is shown in black solid, while the ion (electron) contribution is drawn in red dashed (blue dash-dotted) lines, respectively. Yellow circles denote the neoclassical ion heat transfer rate obtained from the GENE neoclassical solver, red triangles show the ion heat transfer rate (including neoclassical and impurity heat flux), blue pentagons show the electron heat transfer rate, and black diamonds denote the sum of the above contributions. Shaded regions are used to indicate the uncertainty of the interpretive ASTRA values (due to radiation for the total heat transport,

due to equipartition for ion heat transport, and due to both for electron heat transport). Variations of input parameters are explored in Sec. III D.

vative properties of the nonlinearity are ensured by the pseudo-spectral approach.

The simulations have been run with a resolution in parallel spatial $(z)$, parallel velocity $\left(v_{\|}\right)$and magnetic moment $(\mu)$ dimensions of typically $32 \times 32 \times 16$ grid points; the resolutions in radial and toroidal direction were adjusted according to the requirements of each case-explicit values for a number of simulations are listed in Table II.

Note that for the present physical parameters, the radial resolution must be chosen quite large due to the strong magnetic shear, and to a lesser extent also because of the flux tube distortion due to plasma shaping. In addition, we find that it is necessary to include quite small toroidal mode numbers in the simulations, since the fluctuations tend to peak at rather large scales. Namely, for the high-current cases usually a toroidal mode number spacing of $\Delta n=6$ is taken, while for the low current cases $\Delta n=4$ or even $\Delta n=2$ is necessary.

In what follows, the heat transport levels obtained from our simulations will be compared to the experimental values obtained from interpretive ASTRA ${ }^{59}$ modeling, and to the neoclassical ion heat transport obtained from the local GENE neoclassics solver. ${ }^{60}$ The power transported through the full flux surface by species $j$ ('heat transfer rate') is

$$
P_{j}=V^{\prime}\left\langle\boldsymbol{Q}_{j} \cdot \nabla x\right\rangle_{\mathrm{FS}},
$$

where $\nabla x$ is the contravariant basis vector of the radial coordinate, $\langle\cdots\rangle_{\mathrm{FS}}$ denotes a flux surface average, $\boldsymbol{Q}_{j}$ is the heat flux (power per unit area) and $V^{\prime}$ is the derivative of the plasma volume with respect to the $x$ coordinate. For our comparisons, we focus on the electrostatic contribution to $\boldsymbol{Q}_{j}$, generated by the radial component of the fluctuating $E \times B$ drift- the electromagnetic transport channels are found to contribute at most $4 \%$ of the total heat transport, and are thus neglected in the analyzed cases.

\section{Results and analysis}

In Figs. 3a and 3b, the heat transport levels of the simulations marked in boldface in Tab. II are compared to the experimental levels obtained from interpretive ASTRA simulations. The markers show the GENE heat transfer rates (yellow circles: neoclassical; red triangles: ions including neoclassical and impurity; blue pentagons: electrons, black diamonds: sum over the above), while the lines indicate the ASTRA results (red dashed: ion; blue dash-dotted: electron; black solid: sum of both). Note that no contributions due to ETG turbulence are considered in these plots - see Sec. IIIF for simulations and a discussion of ETG turbulence in these discharges.

The simulations shown in Fig. 3a were three-species simulations ( $\# 2$ and $\# 9$ in Tab. II) which included a boron impurity concentration set to match the $Z_{\text {eff }}$ values given in Tab. I. In addition, the assumption $\omega_{n B}=\omega_{n e} / 2$ 
was made for all impurity simulations, as this setting was found to approximately yield

$$
\Gamma_{e} \approx \frac{n_{e}}{n_{D}} \Gamma_{D} \approx \frac{n_{e}}{n_{B}} \Gamma_{B},
$$

whereas using flat impurity profiles $\left(\omega_{n B}=0\right)$ lead to a strong impurity influx, and an increased main ion outflux due to the larger main ion density gradient.

Close analysis of the (nominal parameter) simulations for both radial positions of this discharge revealed that there is a significant amount of particle transport (which would be absent in experiment due to the lack of a core particle source), indicating that the nominal density gradient may be overestimating the actual one to some degree. In Table II, a simulation with slightly decreased density gradient is shown for case $\mathrm{B}$, which gives reduced transport levels.

Examining Figure 3a, we find that using the nominal settings, the simulations of the $0.8 \mathrm{MA}$ discharge moderately overestimate the heat transfer rates by about $35-$ $50 \%$ - the simulations still exhibit an outflow of particles, though, which is responsible for about $20-30 \%$ of the heat transport in case A, and about 20\% (50\%) of the electron (ion) heat transport in case B. A crude correction (neglecting the impact of the density gradient on the driving instabilities) to this result may be obtained by subtracting the convective heat flux $Q_{j}^{\text {conv }}=\frac{3}{2} T_{j} \Gamma_{j}$ from the total value, shifting the heat transfer rates obtained for case A/B to within $20 \%$ of the ASTRA profiles. Additional simulations with input parameter variations for this discharge will be discussed in Sec. III D.

Continuing our analysis with the 0.4 MA discharge, we again find in Fig. 3b that the heat transfer rates obtained from GENE agree within 25-50\% with the ASTRA results. Here, a $50 \%$ underestimation of the combined heat transport levels at the inner radius (case $\mathrm{C}$ ) is found, and a $25 \%$ overestimation at the outer position (case D). The distribution of ion and electron heat transfer rates differs somewhat from mean values ASTRA predicts (more ion heat transport at $r / a=0.75$, less at $r / a=0.85$ ), but are only slightly outside the ASTRA error bars. As will be shown in Sec. IIID, better agreement can be achieved by applying moderate variations of the background gradients.

Importantly, in addition to turbulent heat transport, neoclassical effects also turn out to contribute substantially in the low-current discharge. As the neoclassical transport scales like the squared poloidal ion gyroradius, ${ }^{61}$ the approximate doubling of the safety factor between the two discharges leads to an increase of the neoclassical ion heat transfer rate by a factor of $\sim 4$. At $r / a=0.85$, the neoclassical heat transfer rate thus attains a level of $\sim 0.08 \mathrm{MW}$, approximately equal to the turbulent ion heat transfer rate found for nominal input parameters. Adding both contributions gives a value close to the ASTRA interpreted values. Note that in the GENE simulations of this discharge the predicted particle flux is quite small, as would be expected in the present

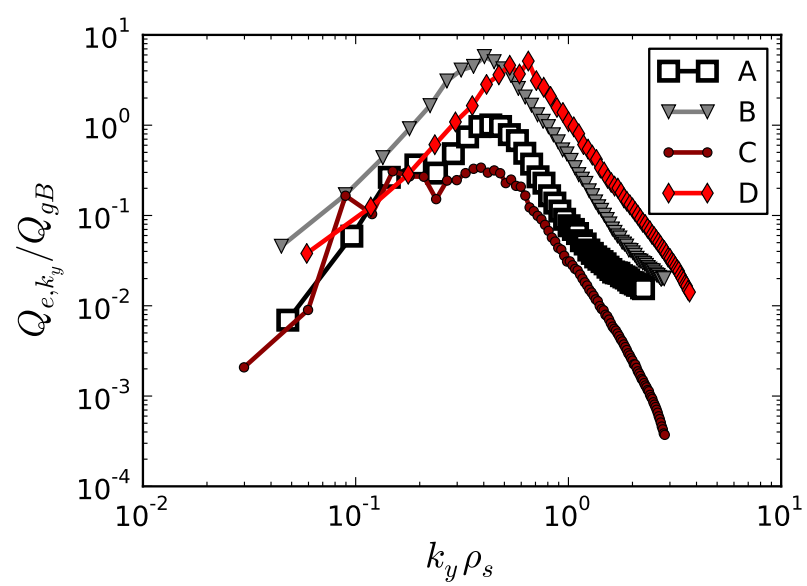

Figure 4: (Color online) Spectra of normalized turbulent electron heat flux for the nominal parameters of all four operation points (cases A-D). The spectra are averaged over time and the radial and parallel direction.

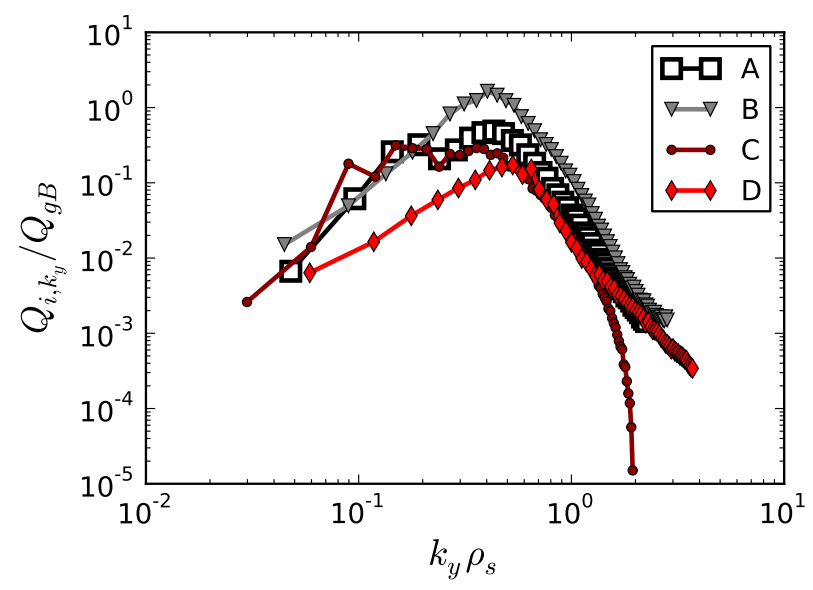

Figure 5: (Color online) Time-averaged spectra of normalized turbulent ion heat flux for the nominal parameters of all four operation points (cases A-D). The spectra are averaged over time and the radial and parallel direction.

ECRH-heated plasmas.

\section{Heat flux spectra}

In Figures 4 and 5, spectra of the electron and ion electrostatic heat fluxes, obtained once again from the boldfaced simulations of Table II, versus the binormal wavenumber are displayed for all operation points discussed so far. Several noteworthy features can be observed: Due to the coexistence of ITG and TEM (or hybrid) instabilities, the spectra of cases $\mathrm{A}$ and $\mathrm{C}$ (i.e., the two $r / a=0.75$ cases) have a rather complex shape exhibiting two peaks, one at low $k_{y}$ due to the ITG mode, 
and a broader one at higher $k_{y}$ due to the TEM mode. The spectra of cases B and D (i.e., of the two outer cases at $r / a=0.85)$ lack the low- $k_{y}$ ITG peak observed at the other two operation points, and exhibit only a single peak around $k_{y} \rho_{s} \sim 0.4$ (case B) and $k_{y} \rho_{s} \sim 0.5-0.6$ (case D).

\section{Comments regarding radial boundary conditions}

We would like to note that, while most of the above simulations could be run straightforwardly with a conventional flux-tube setup ${ }^{39}$ using periodic radial boundary conditions, two-species simulations for case D present an exception. As was hinted already in Sec. IIIB, the presence of unstable microtearing modes at low wavenumbers turned out to lead to problems in nonlinear simulations. More specifically, when running this case in a radially periodic box, typically after a relatively long quasi-stationary phase (lasting for a simulation time of about $300 a / c_{s}$ ) the parallel magnetic potential $\tilde{A}_{\|}$develops structures with large radial extent ("magnetic streamers"), which eventually stretch across the entire radial box, leading to an unbounded growth of fluctuation amplitudes and finally to a blow-up of the simulation.

Two options were successfully explored in order to tackle this problem: First, it is possible to use the global version of the GENE code ${ }^{23}$ (retaining the local approximation), which treats the radial direction in direct space instead of Fourier space and thus allows for Dirichlet-type boundary conditions. Here, the latter assumes fluctuations outside the radial domain to be zero, thereby limiting the maximal extent of turbulent structures. With such a setup, and using appropriate heating terms in order to maintain the background profiles, ${ }^{23,57}$ stable simulations can be achieved, in which the radial extent of the magnetic streamers reaches a steady state. While this approach provides stable simulations, one may question whether it is physically justified to limit the size of turbulent structures in this way. As global effects due to profile variation may impose a natural size limitation to such structures, global simulations could be used to guide the choices made in corresponding local runs - such simulations are very challenging and thus beyond the scope of the present work.

As a second option, the local (periodic) GENE version can be stabilized by adding a small amount of $E \times B$ shear in order to achieve a similar size-limiting effect. Using this approach, an $E \times B$ shearing rate of $\gamma_{E}=0.02 c_{s} / L_{\text {ref }}$ (which is small, but still exceeds the time-averaged experimental value by a factor $\sim 4$ ) is sufficient to achieve a long-term stabilization of the simulation. Both the above approaches were found to yield heat transport levels close to those of a standard periodic simulation before blowup.

In simulations which included boron impurities, microtearing modes were found to be sufficiently weakened to allow for quasi-stationary simulations for at least
$1000 a / c_{s}$, even without any of the above described measures. The GENE result for case D shown in Fig. 3b (\#20 in Table II) was obtained from the quasi-stationary phase of a such a three-species simulation with periodic radial boundaries, without $E \times B$ shear.

\section{Sensitivity with respect to input parameter variations}

In order to estimate the robustness of the present results given experimental profile uncertainties, it is necessary to examine the sensitivity of the simulation results with respect to changes in the plasma parameters. Although the simulations shown in the previous section are relatively expensive (up to $\sim 300,000$ CPUh per run, depending on the number of species and resolution), a number of tests with different resolutions and physics parameters were performed. In Table II, these simulations are listed together with the measured heat transfer rates and the corresponding experimental values.

\section{High-current discharge}

For case A, simulations with nominal parameters (keeping only deuterium and electrons) yielded an overestimation of the experimental electron heat transfer rate by $\sim 40 \%$, and of the ion heat transfer rate by a factor 2.3. The checks performed include a reduction of the radial resolution by a factor of 2 , and the inclusion of compressional magnetic fluctuations. Both these changes had no significant effect on the results. When including boron impurities $\left(n_{B} / n_{e}=3.4 \%\right)$, the saturated transport levels of both heat transport channels are reduced by 20-45\%, thus improving the agreement with experiment.

For case B, with the nominal parameters and two species again an overestimation of the heat transfer rates is obtained, by a factor 3.2 in the electrons, and by a factor 2.9 in the ions. Here, a reduction of the radial resolution can actually lead to false agreement with experiment - both ion and electron heat transfer rates are strongly reduced if only half the radial resolution $\left(n_{x}=\right.$ 256 ) is used (compare simulations $\# 5$ and \#10). Increasing the toroidal mode number spacing from $\Delta n=6$ to $\Delta n=14$ (run \#11) did not further compromise the result. One simulation was performed at even higher resolution, using 1024 radial modes, giving transport levels within $10-20 \%$ of the simulation using 512 modes.

Therefore, using this resolution, several physics parameter variations were performed in order to examine the sensitivity of the transport levels with respect to variations in the input parameters. Since linear simulations indicate that the dominant TEM has a contribution due to density gradient drive (consider also the statements made about the convective heat flux contributions in Sec. III C), the density gradient was reduced by $10 \%$ for run $\# 6$, yielding a considerable decrease in the resulting heat transfer rates, thus reducing the overestimation to 
Table II: Simulation results examining the sensitivity of cases A-D with respect to variation of resolutions and selected physical parameters. The margins of error given for the heat transfer rates indicate the uncertainty of the simulation results due to intermittency.

\begin{tabular}{|c|c|c|c|c|c|c|}
\hline$\#$ & Case+comment & $P_{e} / \mathrm{MW}$ & $P_{i} / \mathrm{MW}$ & $n_{x}$ & $n_{y} \mathcal{N}$ & $\Delta n$ \\
\hline & $A, \exp .(A S T R A)$ & $0.45 \pm 0.21$ & $0.23 \pm 0.06$ & & & \\
\hline 1 & A, nominal & $0.64 \pm 0.09$ & $0.54 \pm 0.09$ & 512 & $48 \quad 10$ & 6 \\
\hline 2 & A+Boron & $0.53 \pm 0.09$ & $0.30 \pm 0.03$ & 512 & 4810 & 6 \\
\hline 3 & A, with $\tilde{B}_{\|}$ & $0.72 \pm 0.11$ & $0.59 \pm 0.11$ & 256 & $48 \quad 10$ & 6 \\
\hline \multirow[t]{2}{*}{4} & A, reduced $n_{x}$ & $0.67 \pm 0.11$ & $0.54 \pm 0.14$ & 256 & $48 \quad 10$ & 6 \\
\hline & B, exp. (ASTRA) & $0.40 \pm 0.30$ & $0.23 \pm 0.06$ & & & \\
\hline 5 & $\mathrm{~B}$, nominal & $1.28 \pm 0.16$ & $0.66 \pm 0.1$ & 512 & $64 \quad 12$ & 6 \\
\hline 6 & $\mathrm{~B}, \omega_{n} \times 0.9$ & $0.92 \pm 0.11$ & $0.48 \pm 0.07$ & 512 & $64 \quad 12$ & 6 \\
\hline 7 & $\mathrm{~B}, \omega_{T e} \times 0.8$ & $0.99 \pm 0.23$ & $0.68 \pm 0.19$ & 512 & $64 \quad 12$ & 6 \\
\hline 8 & B+Boron & $0.77 \pm 0.06$ & $0.24 \pm 0.02$ & 512 & 6412 & 6 \\
\hline 9 & $\mathrm{~B}$, reduced $n_{x}, n_{y}$ & $0.86 \pm 0.09$ & $0.31 \pm 0.03$ & 256 & $48 \quad 12$ & 6 \\
\hline \multirow[t]{2}{*}{10} & $\mathrm{~B}$, reduced $n_{x}, n_{y}$ & $0.75 \pm 0.07$ & $0.28 \pm 0.03$ & 256 & 3228 & 14 \\
\hline & $C, \exp .(A S T R A)$ & $0.70 \pm 0.30$ & $0.29 \pm 0.07$ & & & \\
\hline 11 & $\mathrm{C}$, nominal & $0.47 \pm 0.05$ & $0.52 \pm 0.05$ & 512 & 967 & 2 \\
\hline 12 & C+Boron & $0.25 \pm 0.03$ & $0.24 \pm 0.02$ & 512 & 967 & 2 \\
\hline 13 & C + Boron, $\omega_{T i} \times 1.1$ & $0.44 \pm 0.15$ & $0.47 \pm 0.14$ & 512 & 967 & 2 \\
\hline 14 & C + Boron, $\omega_{T e} \times 1.1$ & $0.27 \pm 0.03$ & $0.31 \pm 0.02$ & 512 & 967 & 2 \\
\hline 15 & C + Boron, $\omega_{T e} \times 1.2$ & $0.50 \pm 0.10$ & $0.39 \pm 0.08$ & 512 & 967 & 2 \\
\hline 16 & $\mathrm{C}$, larger $\Delta n$ & $0.58 \pm 0.11$ & $0.64 \pm 0.14$ & 512 & $48 \quad 12$ & 4 \\
\hline \multirow[t]{2}{*}{17} & $\mathrm{C}$, reduced $n_{x}, n_{y}$ & $0.23 \pm 0.02$ & $0.21 \pm 0.04$ & 256 & $48 \quad 13$ & 4 \\
\hline & $D, \exp .(A S T R A)$ & $0.66 \pm 0.38$ & $0.26 \pm 0.06$ & & & \\
\hline 18 & D, Dirichlet b.c. & $0.57 \pm 0.08$ & $0.10 \pm 0.01$ & 512 & $48 \quad 10.08$ & 4 \\
\hline 19 & $\mathrm{D}, \omega_{T i} \times 1.2$ & $0.55 \pm 0.12$ & $0.24 \pm 0.06$ & 512 & $48 \quad 10.08$ & 4 \\
\hline 20 & $\mathrm{D}, \omega_{T i} \times 1.5$ & $0.45 \pm 0.07$ & $0.37 \pm 0.07$ & 512 & $48 \quad 10.08$ & 4 \\
\hline 21 & $\mathrm{D}$, periodic $+E \times B$ & $0.58 \pm 0.06$ & $0.08 \pm 0.01$ & 512 & 6411 & 3 \\
\hline 22 & D+Boron $($ no $E \times B)$ & $1.10 \pm 0.14$ & $0.06 \pm 0.01$ & 512 & 6411 & 3 \\
\hline 23 & $\mathrm{D}+$ Boron $+E \times B$ & $0.83 \pm 0.08$ & $0.04 \pm 0.01$ & 512 & 6411 & 3 \\
\hline 24 & D + Boron + Dirichlet & $0.64 \pm 0.08$ & $0.04 \pm 0.01$ & 512 & 6416.01 & 4 \\
\hline 25 & $\mathrm{D}$, reduced $\beta=10^{-5}$ & $0.35 \pm 0.03$ & $0.13 \pm 0.02$ & 512 & 6411 & 4 \\
\hline
\end{tabular}

roughly a factor of 2 in both ions and electrons. Reducing the electron temperature gradient $(\# 7)$ lowered the electron heat transfer rate by the same amount, but did not affect the ion heat transport.

Adding a kinetic boron species (using $n_{B} / n_{e}=5.4 \%$ to match the experimental $Z_{\text {eff }}$ ), on the other hand, substantially reduces the transport levels, such that the ion heat transfer rate is matched well and the agreement in the electron heat transfer rate is also improved. Therefore, it may be concluded that through the inclusion of impurities, as well as a small adjustment to the density gradient, agreement with the inferred ASTRA transport levels can be achieved.

\section{Low-current discharge}

Using the nominal parameters for case $\mathrm{C}$ (and two species), nonlinear simulations predict a total heat transfer rate that is in very good agreement with the experimental value, although the distribution between ions and electrons differs from the ASTRA interpreted values. Nevertheless, since impurities lead to a substantial transport reduction in cases $\mathrm{A}$ and $\mathrm{B}$, their influence consequently must be studied for the $0.4 \mathrm{MA}$ discharge as well.

Running the case $\mathrm{C}$ parameters with a boron density of $n_{B} / n_{e}=5.0 \%$ (again chosen to match the experimental $Z_{\text {eff }}$ through boron alone), both the ion and electron heat transfer rates are reduced by factors of $\sim 2$ (see \#12), thus underestimating the mean ASTRA levels by roughly this factor, and is slightly below the ASTRA error bars 


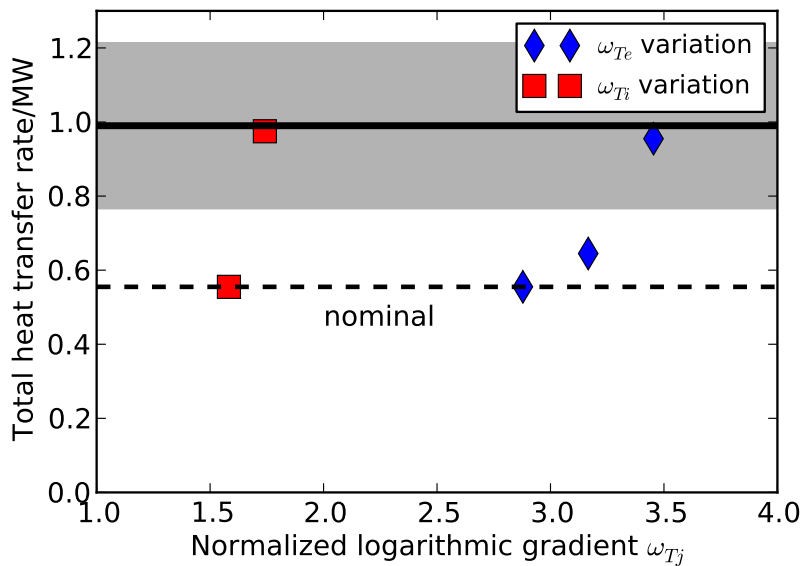

Figure 6: (Color online) Total heat transfer rates (including neoclassical) for variations of ion (red squares) and electron (blue diamonds) logarithmic gradients $\omega_{T j}$ from their nominal values (indicated by dashed line) in case $\mathrm{C}$. With moderately increased values, the ASTRA heat transport levels (solid line, with error band) can be matched.

as well. For this reason, additional simulations were performed (see Fig. 6), increasing either the electron or ion temperature gradient by $10 \%$ (runs \#13 and \#14, respectively). The electron temperature profile was found to be moderately stiff, yielding a $30 \%$ increase of $P_{e}$ with $10 \%$ increased electron temperature gradient. With slightly increased ion temperature gradient, on the other hand, it was very difficult to obtain a steady saturation level over the simulation time of $\sim 750 L_{\text {ref }} / c_{s}$ - the average heat transfer rates obtained during that simulation time recover approximately the reduction found before when adding impurities. Similar statements apply to the simulation $\# 15$, which was run with $20 \%$ increased electron temperature gradient. From the above results we can conclude that agreement of the transport levels within the errors can be achieved, once again considering small variations of the input parameters.

Numerical convergence of the above simulation results was examined by reducing perpendicular resolutions for the nominal parameters (runs \#16 and \#17). As for case $\mathrm{B}$, we find little change when increasing the toroidal mode number spacing $\Delta n$, but a strong reduction of heat transport levels when lowering the radial resolution to 256 modes.

Finally, we turn to case D, which proved to be the most delicate parameter set in terms of numerical stability, as was already mentioned in Sec. III C. The nominal parameters were therefore run with Dirichlet boundary conditions (run \#18), using Krook-type buffer zones acting on all $k_{y}$ modes close to the boundary (see Ref. 23), as well as Krook-type heat and particle sources. ${ }^{57}$ Using this setup, the electron heat transfer rate is close to the ASTRA result. The turbulent ion heat trans- fer rate, on the other hand, underestimates the ASTRA value somewhat-note, however, that according to the GENE neoclassical solver, the neoclassical heat transfer rate is expected to largely make up for the discrepancy, adding approximately $0.08 \mathrm{MW}$ to the balance at this radial position. When increasing the driving ion temperature gradient by $20 \%$, the turbulent ion heat transfer rate is raised to $0.24 \mathrm{MW}$ (see run \#19), which together with neoclassics, also surpasses the mean ASTRA value. Furthermore, increasing for a test the ion temperature gradient by $50 \%$ (run \#20) leads to an ion heat transfer rate of 0.37 MW, which exceeds, together with neoclassical levels, the mean ASTRA level by more than 70\%. Therefore, turbulent and neoclassical contributions match the experimental levels for a moderate gradient increase of $10-20 \%$.

When using, instead of the Dirichlet approach, a periodic (flux-tube) simulation with an $E \times B$ shearing rate of $\gamma_{E}=0.02 c_{s} / a$ (compare run $\# 21$ to $\# 18$ ), very similar heat transfer rates are obtained. Adding kinetic boron impurities (\#22, again assuming $\omega_{n B}=\omega_{n e} / 2$ to retain close-to-zero particle flux, and $n_{B} / n_{e}=6.5 \%$ ) yields a substantial increase of the electron heat transfer rate (now overestimating the mean ASTRA result by almost $70 \%$ ), and a further slight reduction of the ion heat transfer rate. Running the same setup either with Dirichlet boundaries or with $E \times B$ shear $(\# 23$, \#24) generates somewhat reduced electron heat transfer rates, within the ASTRA error bars, while ion heat transfer rates remain small at about $0.04 \mathrm{MW}$.

Finally, running again nominal parameters (with no impurities), but with an artificially reduced plasma beta of $\beta_{e}=10^{-5}$ (run $\# 25$ ), the microtearing instability is stabilized enough to achieve a numerically stable simulation without resorting to Dirichlet boundaries or $E \times B$ shear, giving at the same time a slightly larger ion heat transfer rate. However, a $40 \%$ reduction in electron heat transfer rate is observed compared to the nominal run, indicating an electromagnetic enhancement of electron heat transport levels in this case, despite the predominantly electrostatic nature of the driving instabilities.

Summarizing the present sensitivity study, we do not observe any systematic underprediction of heat transfer rates in our gyrokinetic simulations. Even for nominal parameters, the GENE transport level predictions are not systematically outside the error bars of the ASTRA predictions, and could be matched using reasonable variations of the input parameters. For the same discharges studied here, however, it was recently shown ${ }^{41}$ that quasilinear TGLF results do substantially underestimate (by a factor 3-5) the heat transfer rates for the 0.4 MA discharge, while agreeing better with the $0.8 \mathrm{MA}$ discharge. Our results therefore suggest that the shortfall of the TGLF transport predictions in this case is likely related to the assumptions made in the model rather than to a breakdown of the underlying gyrokinetic theory. This question will be further addressed in Sec. IV. First, the GENE simulations are examined more closely to estab- 
lish whether quasilinear modeling is applicable at all for the relatively cold L-mode plasmas studied here.

\section{E. Analysis and comparison of linear and nonlinear fluctuation characteristics}

In the debate about underpredicted transport levels in L-mode turbulence, a recurring topic has been the question whether the problem might be caused by an illmodeled transition from plasma core turbulence, which often retains features (cross phases, mode frequencies) of the driving linear instabilities, to edge turbulence, which has been reported to exhibit little correspondence to linear modes. ${ }^{18,19,62}$

\section{Cross phase analysis}

Having shown that in the present GENE simulations we do not find any systematic transport underprediction, we will now demonstrate that even at the outermost positions studied here, many turbulence characteristics remain essentially attached to those of the linear instabilities. To this end, we compare the phase shift $\vartheta$ between the fluctuating electrostatic potential $\tilde{\phi}$ and fluctuations of the perpendicular temperature $\tilde{T}_{\perp, i}, \tilde{T}_{\perp, e}$, which is obtained by calculating at the outboard midplane for each $k_{y}$ mode

$$
\begin{array}{r}
\left\langle\vartheta\left[\tilde{\phi}, \tilde{T}_{\perp, j}\right]\right\rangle_{k_{x}}\left(k_{y}\right) \\
=\frac{\sum_{k_{x}} \arg \left[\tilde{\phi}\left(k_{x}, k_{y}\right) / \tilde{T}_{\perp, j}\left(k_{x}, k_{y}\right)\right] W\left(k_{x}, k_{y}\right)}{\sum_{k_{x}} W\left(k_{x}, k_{y}\right)}
\end{array}
$$

where $W\left(k_{x}, k_{y}\right)=\left|\tilde{\phi}\left(k_{x}, k_{y}\right)\right|\left|\tilde{T}_{\perp, j}\left(k_{x}, k_{y}\right)\right|$ is an amplitude-dependent weighting factor, $\langle\cdots\rangle_{k_{x}}$ indicates an average over all $k_{x}$ modes, and $j$ is the species index.

In Figs. 7 and 8 , the phase shifts of $\tilde{\phi}$ vs. $\tilde{T}_{\perp, e}$ and $\tilde{T}_{\perp, i}$ (of the main ions) are shown for the simulations of cases A-D, using nominal parameters, but including boron impurities (i.e. runs $\# 2, \# 8, \# 12, \# 21$ ). In Fig. 9, furthermore, the same phase shifts are displayed for a simulation of the additional flux surface $r / a=0.9$ of the $0.4 \mathrm{MA}$ discharge ${ }^{63}$ (neglecting impurities here).

While the shifts between $\tilde{\phi}-\tilde{T}_{\|}$and $\tilde{\phi}-\tilde{n}$ are relevant as well for electrostatic heat transport, the qualitative statements made below for $\tilde{\phi}-\tilde{T}_{\perp}$ are valid also for the other variable pairs, but not shown here for brevity. In the figures, the colored contours display the crossphases obtained from the saturated phase of nonlinear simulations (the ones marked in boldface in Tab. II), while the red squares (green circles) are used to display the cross phases of the linear instability with the largest (second largest) growth rate. The transitions between dominant modes, finally, are marked by dashed lines, and the labels indicate the dominant mode in each region.
Several observations can be made from these plots. Generally, the agreement between linear and nonlinear cross phases is remarkably good even for the most extreme parameters studied here (i.e. the outermost positions of the low-current discharge), especially in the wavenumber regime most important for transport, $0.1 \lesssim$ $k_{y} \rho_{s} \lesssim 0.5$. Moreover, it can be observed that the nonlinear cross phases do tend to trace those of the mostunstable linear mode - see Figs. 8a for a case in which the nonlinear $\tilde{\phi}-\tilde{T}_{\perp, i}$ cross phase (which determines the ion heat flux) at high $k_{y}$ 'prefers' to follow that of the more unstable hybrid/TEM instability rather than that of the ITG mode. On the other hand, knowledge of the subdominant instabilities does provide additional useful information in cases like Fig. $8 \mathrm{c}$, where the $\tilde{\phi}-\tilde{T}_{\perp, i}$ cross phase agrees with the subdominant ITG rather than the dominant ETG mode, or in the mode transition regime of Fig. 8d, where the nonlinear cross phase averages over those of the two coexisting linear modes.

In the cases studied here, the analysis of the $\tilde{\phi}-\tilde{T}_{\perp, e}$ cross phase, relevant for electron heat transport, does not benefit much from knowledge of the subdominant modes, as both the dominant and subdominant instabilities place that phase shift close to the resonant $\pi / 2$ value, as do the turbulence simulations. Microtearing modes do not contribute substantially in the present analysis since only electrostatic phase shifts are studied, while MTMs generate almost exclusively electromagnetic transport, characterized mainly by the cross phase between the parallel electron heat flux and the radial magnetic field fluctuations. ${ }^{55,64}$

\section{Frequency analysis}

In addition to the above cross phase analysis, a comparison between linear and nonlinear drift frequencies can serve to quantify the degree to which linear and nonlinear physics overlap. Here, as an example we perform this comparison for case D. From a nonlinear simulation, we extract the real frequencies of the electrostatic potential fluctuations by applying a windowed-FFT method to the simulation data, and plot in addition the mode frequencies obtained from the linear eigenvalue analysis. The result of this comparison is plotted in Fig. 10, and again displays very good agreement between linear and nonlinear regimes. Here, one may note that the wavenumber range in which the ITG/TEM frequencies can be identified in fact extends beyond the range where each individual mode dominates (red squares vs. green circles), so that both frequencies coexist between $k_{y} \rho_{s} \approx 0.2-0.4$, similar to the observations made in Ref. 65. The double peak visible in the frequencies of the nonlinear regime at $k_{y}=0, \omega \approx 0.5 c_{s} / L_{\text {ref }}$ corresponds to a GAM oscillation (whose frequency analytically scales as ${ }^{66} \omega_{G}=\sqrt{2} c_{s} / R \approx 0.55 c_{s} / L_{\text {ref }}$ in circular plasmas), which can be found also in linear GAM simulations with $k_{x} \rho_{s} \approx 0.2$. The microtearing modes observed 


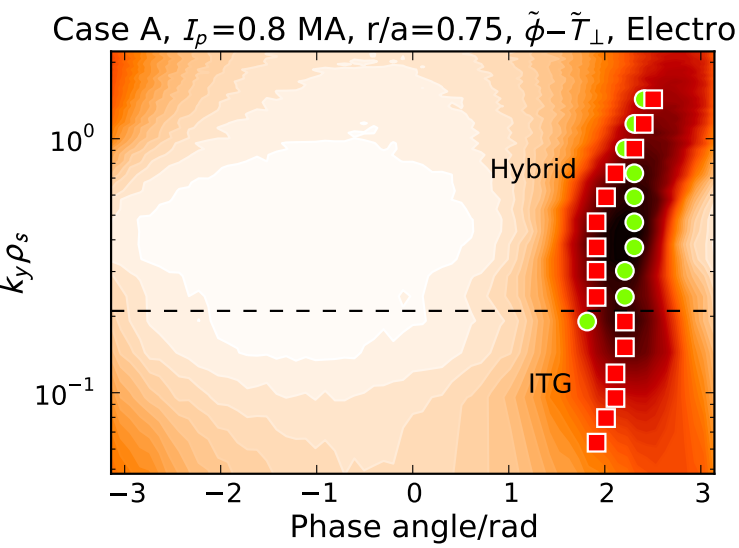

(a)

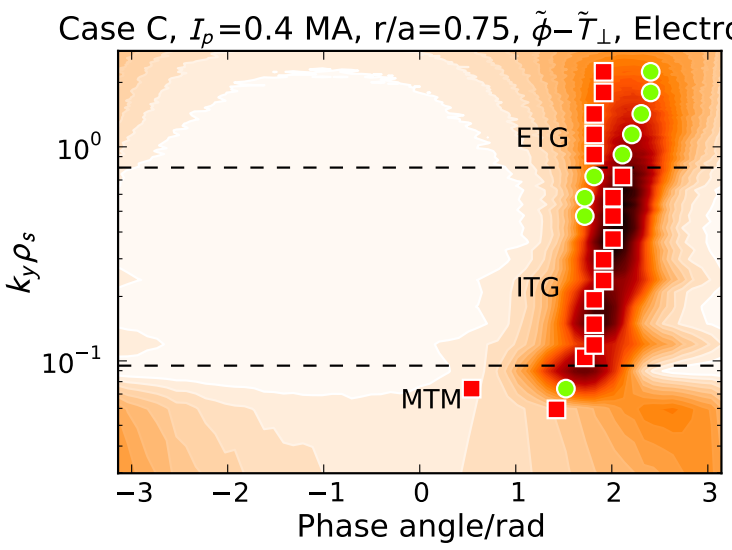

(c)

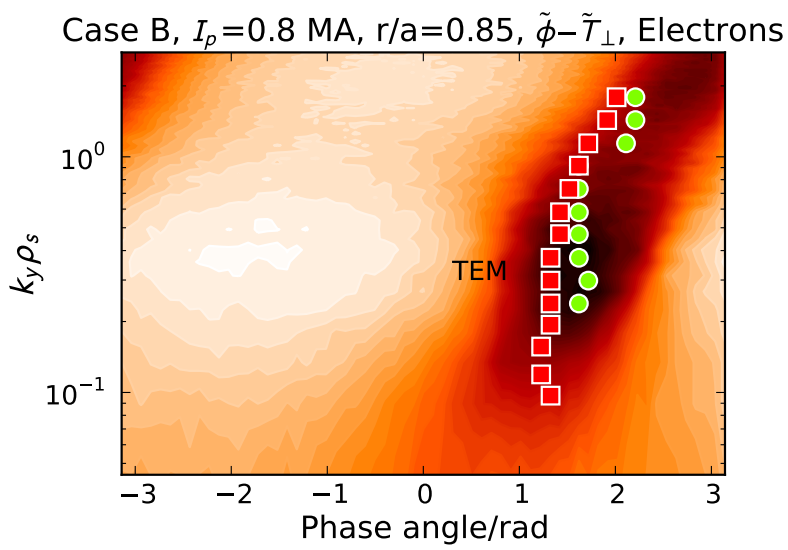

(b)

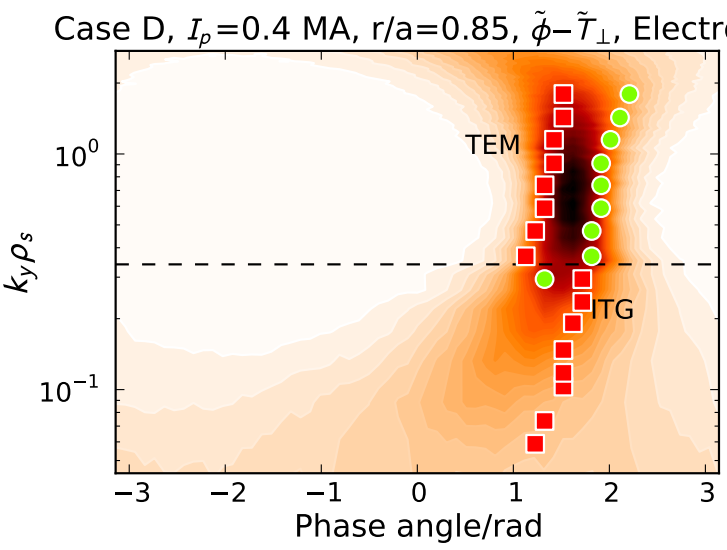

(d)

Figure 7: (Color online) Comparison of linear (markers) and nonlinear (contours) cross-phases between $\tilde{\phi}$ and $\tilde{T}_{\perp e}$, where amplitudes increase from white to black. The most unstable linear modes are plotted as red diamonds, the second-most unstable modes as green circles, and dashed lines separate wavenumber regions dominated by different instabilities.

in the linear simulations of Sec. IIIB are weakened in the simulations which include impurities, so that they do not appear in the frequency spectra. Even in nonlinear simulations without impurities, their influence on the spectra is weak - this is likely due to the fact that the spectra have been generated from electrostatic potential data. Measuring instead frequencies on the electromagnetic $A_{\|}$potential would provide stronger microtearing signals, but such an analysis is not shown here.

To summarize the findings of this section, one can state that there appears to be a solid foundation for quasilinear modeling of the present L-mode discharges out to $r / a=0.9$, at least. It should be kept in mind, though, that in this case the observed agreement between linear and nonlinear characteristics was found here using full gyrokinetic simulations, which contain more complete physics than the simplified (but much faster) quasilinear transport models currently in use (see, e.g. Refs. 10 and $67)$. It is therefore not self-evident that these models will straightforwardly obtain the same results in their present form.

\section{F. Contributions due to ETG turbulence}

When attempting to match experimental transport levels in gyrokinetic simulations, it is important to keep in mind that when comparing the simulated electron heat transfer rates to experimental data, the contributions due to electron scale turbulence (usually by ETG modes) should be examined as well. A recent study ${ }^{9}$ has begun work on L-mode discharges of the Alcator-C-Mod tokamak, and found cases where indeed the contribution of ETG turbulence to $Q_{e}$ constituted a significant fraction of the electron heat transport. In the present section, we therefore analyze the properties of electron scale turbulence in the discharges studied above.

In Sec. III B, it was found that the low-current dis- 


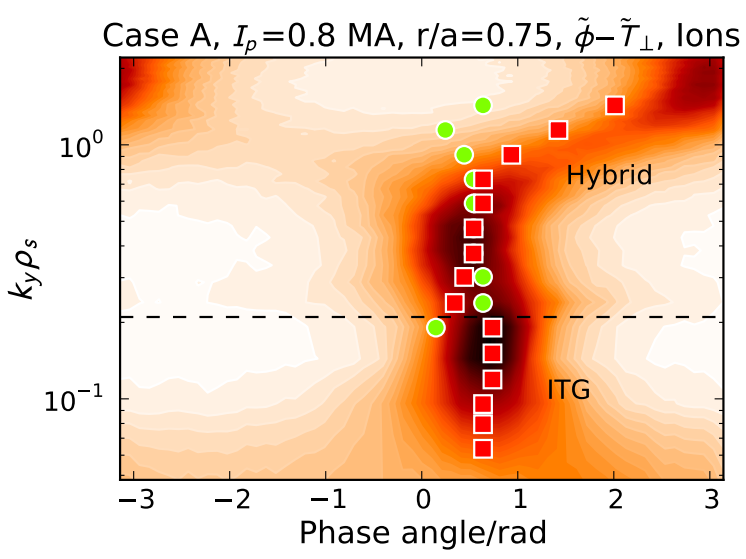

(a)

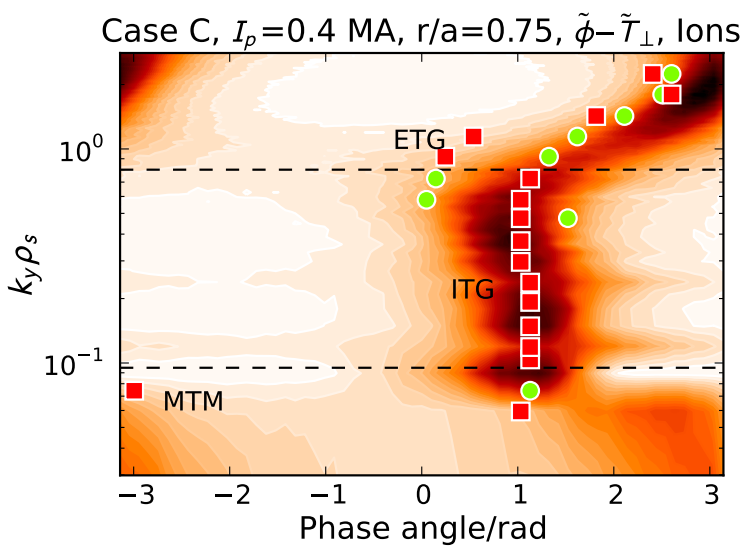

(c)

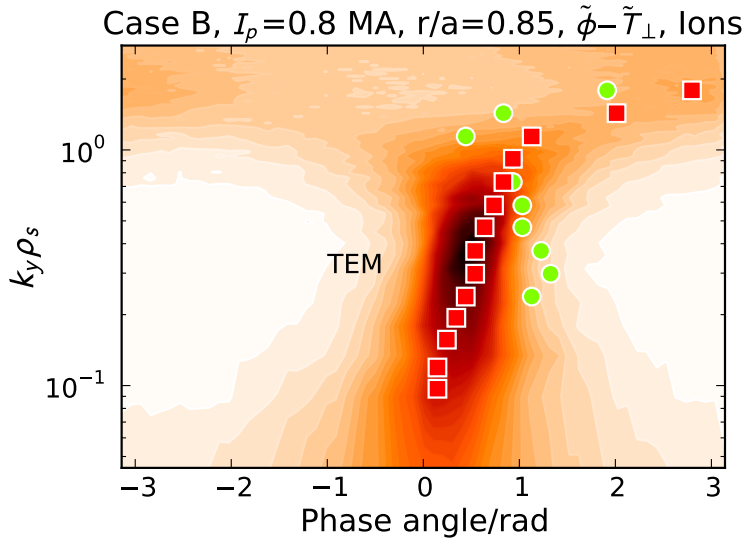

(b)

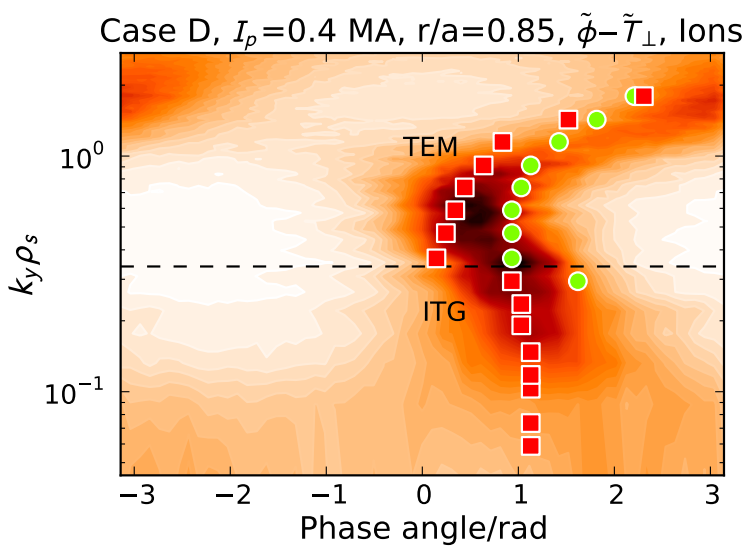

(d)

Figure 8: (Color online) Comparison of linear (markers) and nonlinear (contours) cross-phases between $\tilde{\phi}$ and $\tilde{T}_{\perp i}$, with amplitudes increasing from white to black. The most unstable linear modes are plotted as red diamonds, the second-most unstable modes as green circles, and dashed lines separate wavenumber regions dominated by different instabilities.
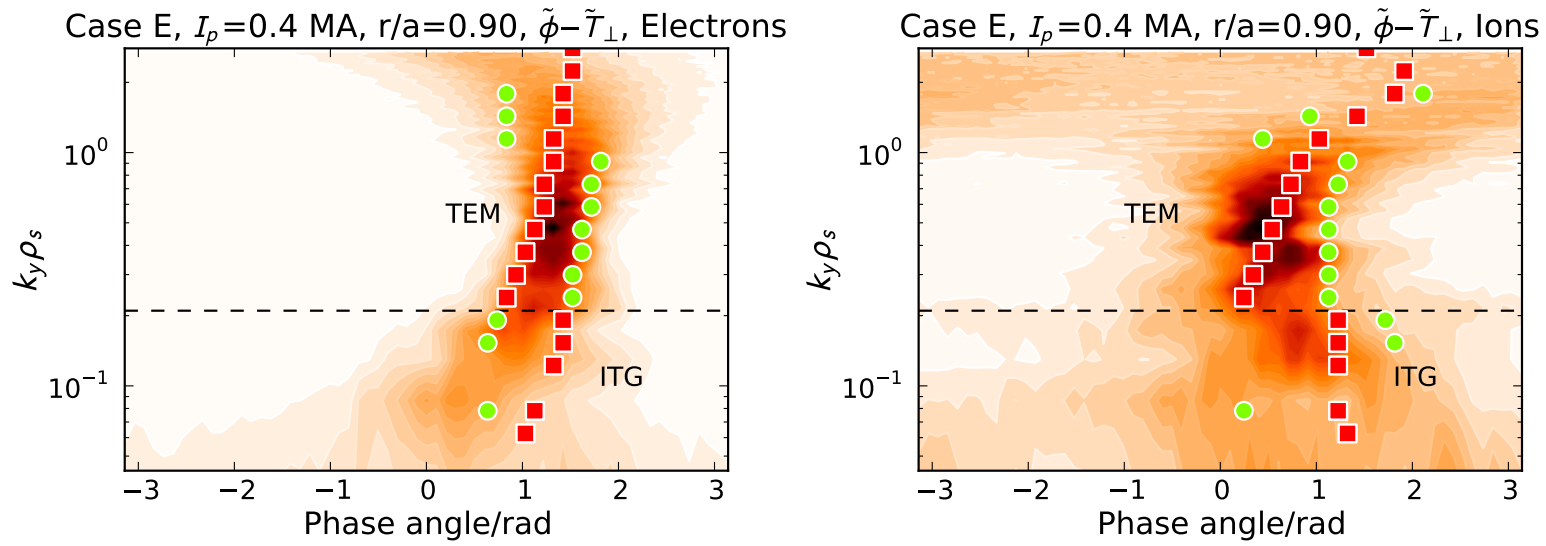

Figure 9: (Color online) Comparison between linear and nonlinear cross phases for $r / a=0.9$ of the $0.4 \mathrm{MA}$ discharge. The colors and labels are the same as those of Figs. 7 and 8 . 


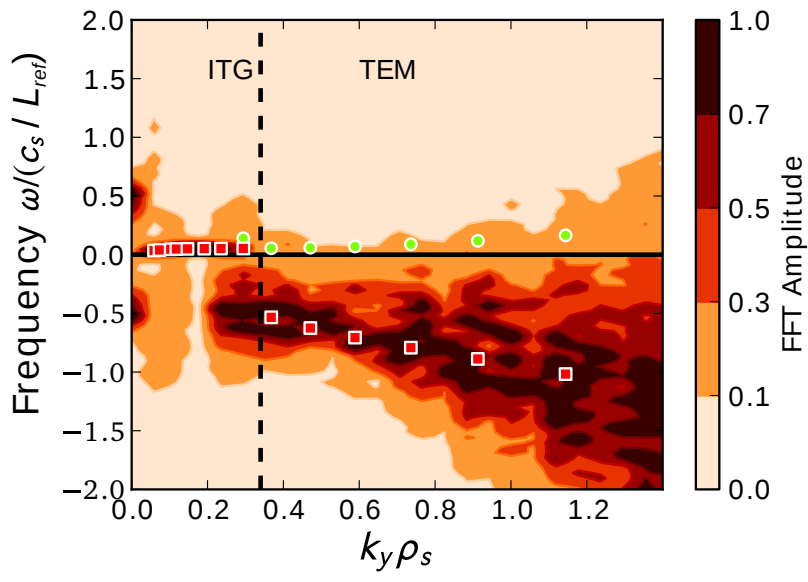

Figure 10: (Color online) Real frequencies obtained by FFT analysis of the nonlinear simulation data for the $r / a=0.85$ position of the low-current discharge (case

D). The linear mode frequencies are plotted as red squares (green circles) for the dominant (subdominant) instability.

charge (cases C and D) had relatively stronger ETG instabilities than the high-current discharge (cases A and B), implying that ETG turbulence may contribute measurably in the former. For that reason, results from dedicated ETG simulations of these two cases are discussed in the present section. Before examining the simulation results, we analyze the discharge data from a linear point of view by calculating the critical gradients of the ETG instabilities, using the formula of Ref. 68,

$$
\begin{aligned}
R / L_{T, \text { crit }}=\max \left[\left(1+Z_{\mathrm{eff}}\right.\right. & \left.\frac{T_{e}}{T_{i}}\right)\left(1.33+1.91 \frac{\hat{s}}{q}\right) \\
& \left.\times\left(1-1.5 \frac{r}{R}\right), 0.8 \frac{R}{L_{n}}\right] .
\end{aligned}
$$

For this analysis, we convert the data from Table I from $\rho_{\text {tor }}$ to $r$ coordinates (all necessary geometric quantities can be found in the Table), and convert the resulting critical gradients back to the convention employed here. This procedure yields for cases A-D, respectively, critical gradients of $\omega_{T e \text {,crit }}=2.14,2.02,1.51$, and 1.21. Thus, the observation of more pronounced ETG instabilities in cases C and D (see Sec. III B) can be traced back to a reduced linear threshold due to the larger safety factor values - note, however that the linear simulations shown in that Section did not include impurities, in contrast to the critical gradient values calculated here. Even though impurities tend to raise the linear threshold gradient for ETG instabilities by increasing $Z_{\text {eff }}$, the experimental gradients exceed the threshold in all four cases. In addition to the lower thresholds of cases $\mathrm{C}$ and $\mathrm{D}$, the ratio $\eta_{e}=L_{n e} / L_{T e}$ (where $L_{n e}, L_{T e}$ are density/temperature profile scale lengths) driving the ETG instabilities is also considerably larger in those cases, reaching values of about 3.5 to 4 , while in cases $\mathrm{A}$ and $\mathrm{B} \eta_{e} \approx 2$.
In order to study the actual transport contributions due to ETG turbulence, we now turn to nonlinear simulations for all cases $\mathrm{A}-\mathrm{D}$, focusing exclusively on electron scales. Since any possible coupling between ion and electron scales ${ }^{52}$ is ignored with this approach, such simulations of pure ETG turbulence pose difficulties of their own: In toroidal plasmas, ETG turbulence often has the property of generating extremely elongated radial structures (streamers ${ }^{21,69,70}$ ), requiring large radial simulation domains. In reality, however, turbulence at larger scales (neglected here) would generate a fluctuating background, which may act in a stabilizing way on the smaller ETG structures and thus limit their radial extent. $^{52,71,72}$

In order to model this stabilizing effect, we add a weakly sheared background flow, as discussed already in Sec. III C, where MTMs caused saturation problems for the case D simulations. Setting the shearing rate to values much smaller than the linear growth rate $\left(\gamma_{E} \sim\right.$ $\left.0.002 L_{\mathrm{ref}} / v_{t e}\right)$ is found to be sufficient here to prevent the formation of large streamers, while at the same time having only a moderate effect on the resulting transport levels.

Using this setup, simulations with an adiabatic ion response (including impurities through the parameter $\tau=Z_{\text {eff }} T_{e} / T_{i}$, see Ref. 68) were performed for each of the four cases. Despite the small $E \times B$ shearing rate used in these simulations, the heat transfer rates generated were rather small, attaining values of $0.06 \mathrm{MW}$, $0.07 \mathrm{MW}, 0.04 \mathrm{MW}$, and $0.09 \mathrm{MW}$, respectively, for cases A-D. When neglecting impurities, on the other hand, the transport levels rise to $0.25 \mathrm{MW}$ for case $\mathrm{C}$, and $0.41 \mathrm{MW}$ for case D. It is therefore essential to retain their contribution in order to obtain a realistic picture.

In summary, the ETG transport levels obtained in this Section do not alter the statements of Sec. III C substantially, as they present only small additions to the electron heat transport found in ion scale simulations. We would like to stress here, though, that this statement does not apply to L-mode discharges in general. For instance, as can be seen in Table I, the well-known DIII-D 'shortfall' discharge 128913 exhibits both a lower $Z_{\text {eff }}$, as well as a larger $T_{i} / T_{e}$ ratio than any of the cases examined here. Thus, we have $\tau \approx 1$ in that case, while all of the ASDEX Upgrade operation points studied here have $\tau>2$. Calculating the linear threshold gradient for DIII-D yields $\omega_{T e, \text { crit }}=1.10$, which is strongly exceeded by the experimental gradients, while at the same time $\eta_{e}=4.6$. More significant ETG contributions can thus be expected in the DIII-D L-mode discharge. Finally, it should be noted that, by the temperature fluctuations due to large-scale turbulence can also locally destabilize ETG turbulence, which may thus add to the electron heat transport. These effects can only be conclusively examined in simulations encompassing both ion and electron gyroradius scales, though, which are outside the scope of this paper. 


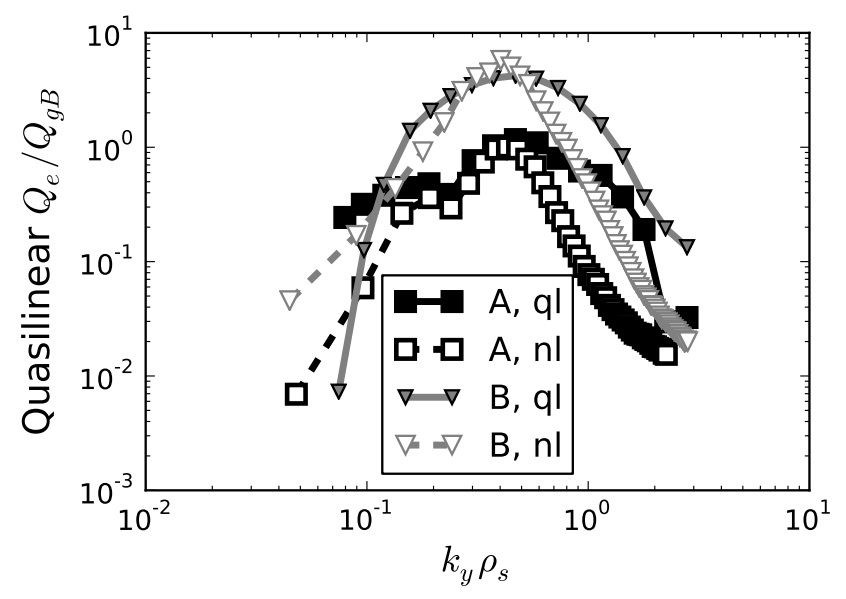

Figure 11: Comparison of electron heat flux predicted by the quasilinear model of Eq. 3 (solid lines, full symbols) against the nonlinear results (dashed lines, open symbols) for cases A and B.

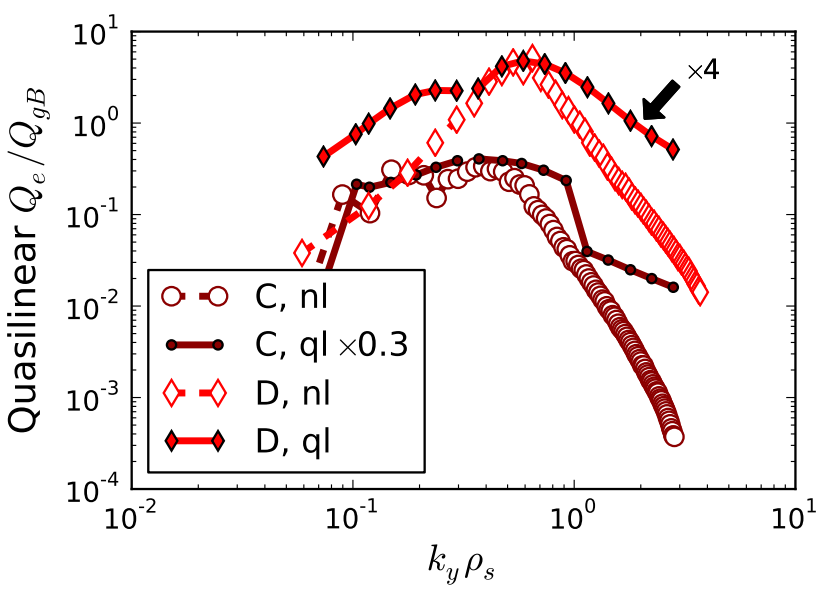

Figure 12: (Color online) Comparison of electron heat flux predicted by the quasilinear model of Eq. 3 (solid lines, full symbols) against the nonlinear results (dashed lines, open symbols) for cases C and D.

\section{CONSEQUENCES FOR QUASILINEAR MODELING}

As has been demonstrated in the previous sections, there does not appear to be any fundamental obstacle for quasilinear modeling of colder L-mode regions. In the present section, we therefore discuss the reasons for the apparent failure of present quasilinear models in some of these cases (documented, e.g. in Refs. 4, 6, 7, and 41 ), and we give some suggestions for the improvement of such models.

Quasilinear transport modeling basically rests on two pillars. The first consists of a more or less elaborate approach to describing the physics of linear instabilities for given plasma parameters - these usually involve solving linearized gyrofluid or simplified gyrokinetic equations.
Already at this level, it is not guaranteed that all relevant effects are captured in such models, since linear instabilities can develop quite complex mode structures in simulations which consider strong shaping. ${ }^{27}$ However, it must be stressed here that even from the most accurate linear physics description it is not possible to deduce any information on nonlinear saturation levels. Thus, the transport levels remain entirely unspecified, and can only be found reliably by actually solving the full nonlinear equations.

Due to this fact, as the second pillar of any quasilinear transport model, an empirical nonlinear saturation rule must be employed to be able to deduce transport levels from the linear results. Such a rule usually consists of an assumption on the shape of the transport spectrum (or the saturated electrostatic potential) and a saturation amplitude, which is derived, e.g., from mixing length estimates ${ }^{67}$ or obtained by fitting an ad-hoc formula to nonlinear simulations. ${ }^{11}$ Present quasilinear models have mainly been benchmarked/calibrated to simulations of core plasma turbulence - saturated turbulence levels outside this parameter regime are therefore likely to deviate from the model predictions.

For core parameters, turbulent diffusivities are usually found to be of the order of the gyro-Bohm diffusivity $\chi_{g B}=\rho_{s}^{2} c_{s} / L$ (where $L \in\left\{a, R, L_{n}, L_{T e}, L_{T i}\right\}$ ). In the cold outer core and edge of L-mode plasmas, however, due to the scaling $\chi_{g B} \propto T^{3 / 2}$, the gyro-Bohm heat flux decreases like $Q_{g B} \propto T^{5 / 2}$, so that models fitted to core turbulence amplitudes will almost inevitably fail unless their saturation rules are adapted or extended for L-mode parameters, which require saturation at much higher amplitudes than in the hot core of $\mathrm{H}$-mode plasmas. For the operation points examined in the present work, e.g., $Q_{g B}$ decreases roughly by a factor of $4(2.5)$ in the 0.8 (0.4) MA discharge, going from $r / a=0.75$ to $r / a=0.85$, while the heat flux values obtained from the nonlinear gyrokinetic simulations accordingly rise from $Q_{e} / Q_{g B} \sim 10$ to $Q_{e} / Q_{g B} \sim 50(40)$ - visible also in the heat flux spectra of Figs. 4 and 5.

As a simple test, we evaluate quasilinear diffusivities for the present ASDEX Upgrade cases A-D, using the quasilinear model proposed in Ref. 22, which was successfully applied therein to pure TEM turbulence. This model estimates saturated quasilinear diffusivities by evaluating

$$
\chi_{Q L}=\frac{\gamma}{\left\langle k_{\perp}^{2}\right\rangle},
$$

where

$$
\left\langle k_{\perp}^{2}\right\rangle=\frac{\int_{-m \pi}^{m \pi} k_{\perp}^{2}\left|\phi_{k_{y}}(z)\right|^{n} \mathrm{~d} z}{\int_{-m \pi}^{m \pi}\left|\phi_{k_{y}}(z)\right|^{n} \mathrm{~d} z}
$$

is the parallel average (extending over $m-1$ poloidal turns) of the perpendicular wavenumber, weighted by a power $n$ of the electrostatic potential, and $\gamma$ is the linear growth rate. For each $k_{y}$, the diffusivity $\chi_{Q L}$ is taken 
to be the satured diffusivity of the dominant heat transport channel - the diffusivities for the other species are then computed according to the ratios of the transport quantities evaluated from the linear simulations.

Using this scheme and setting $n=2$ and $m=15$, we calculate spectra of the quasilinear prediction for the electron heat flux $Q_{e} / Q_{g B}$, which are plotted in Figs. 11 and 12. The former figure shows that, without applying any scaling factors, for the high current discharge the quasilinear model predictions agree surprisingly well with the nonlinear results in terms of the peak position and amplitudes of the heat flux spectra. Also, the double peak structure found in case A agrees well with the nonlinear simulation results.

For cases $\mathrm{C}$ and $\mathrm{D}$, on the other hand, the predictions of the quasilinear model cannot be used as straightforwardly. In Fig. 12, the quasilinear spectrum for case $\mathrm{C}$ has been multiplied by a factor 0.3. Apart from the amplitude, the shape of the spectrum, and the peak position still agree quite well with the nonlinear result. In contrast, for the parameters of case $\mathrm{D}$, we find that the model does not handle the coexistence of ITG and TEM modes well. In order to make the peak amplitudes agree, the heat flux predictions for the TEM branch (marked in the figure) had to be multiplied by a factor 4 . The ITG fluxes, on the other hand, were not rescaled, but overestimate the nonlinear results by a similar factor. The quasilinear model of Eq. (3) therefore clearly needs refinement to enable a reliable treatment of such cases, possibly along the lines of Ref. 65, where similar cases with coexisting ITG/TEM instabilities have already been treated. Such a study is beyond the scope of the present paper, though.

In summary, while the results of Sec. IIIE imply that quasilinear modeling should be in principle applicable to the outer core of L-mode plasmas, we would like to stress that to enable reliable predictions for such regimes, significant extensions to the models presently in use are likely required. In particular, the empirical saturation rules used in current quasilinear models should be benchmarked/fitted to nonlinear gyrokinetic simulations which simultaneously include large gradients, large safety factor, strong magnetic shear, and strong shaping. Furthermore, collisional and electromagnetic effects, which are more important in the outer core of L-modes, should be included. We are aware that this requires an enormous computational effort, as is obvious also from the present work, but in our view this is a necessary step in order to achieve a reliable quasilinear modeling of L-mode plasmas.

\section{v. CONCLUSIONS}

The study presented in this paper focused on nonlinear gyrokinetic simulations of turbulence in the outer region of L-mode plasmas, using the GENE code. A seemingly systematic underestimation of transport levels in DIII-D
L-mode plasmas, which was reported in several publications in recent years, motivated the present study of turbulence in ASDEX Upgrade L-mode discharges. In our simulations, no evidence of a systematic underprediction could be found - instead, even using the nominal experimental plasma parameters, our most comprehensive simulations predicted transport levels close to the inferred experimental values. Even better agreement could be achieved through moderate variations of input parameters around their nominal values. Conversely, for prescribed fluxes, as is in principle possible with transport solvers like TRINITY ${ }^{73}$ or TGYRO ${ }^{74}$, one would obtain quite accurate profile predictions for the analyzed cases. However, performing extensive parameter variations with nonlinear gyrokinetic simulations is very expensive, since the plasma conditions occurring in the outer regions of L-mode plasmas place severe constraints on the resolutions and physics content to be retained. This study thus involved a large computational effort, amounting to about 15 million CPU-hours in total - attempts to exactly match experimental transport levels for many different operation points therefore constitute an immense undertaking.

With the nonlinear simulation data gathered in this study, an extensive comparison of cross phases and frequencies with those obtained in linear simulations was performed, which revealed that even in conditions of cold L-mode plasmas, turbulence properties like the cross phases and drift frequencies resemble closely those of the driving instabilities, at least out to a radial position of $r / a=0.9$. Hence, the applicability of quasilinear models for such plasmas is, in principle, confirmed.

The problems of current quasilinear models in matching the experimental fluxes therefore appear to stem not from a failure of the underlying gyrokinetic theory, but mainly from limitations of the empirical nonlinear saturation rules which link linear physics to transport levels. In the cold L-mode edge, saturation amplitudes necessarily must be larger in order to overcome the degradation of gyro-Bohm transport levels with temperature. Considering the fact that the existing models are calibrated largely to the saturation levels of electrostatic core turbulence simulations, it is clear that an additional effort is required to devise saturation rules which apply also to colder L-mode plasmas with simultaneously occurring strong magnetic shear, large safety factor, strong shaping, and steep gradients.

The underpredictions found even in nonlinear gyrokinetic simulations of certain L-mode plasmas are not confirmed for the discharges and physical/numerical setups used in the present paper. In addition, close agreement between the GENE and GKW gyrokinetic codes was demonstrated. A substantial international benchmarking effort is currently underway to address the question whether this 'shortfall' problem is caused by numerical issues and/or connected to certain discharge conditions. 


\section{ACKNOWLEDGMENTS}

The authors would like to thank K. Lackner, C. Holland, D.R. Mikkelsen, and C. Angioni for fruitful discussions. The computations for the present study were carried out in part on the Helios computer of the IFERCCSC. Furthermore, we acknowledge PRACE for awarding us access to the SuperMUC computer based at the Leibniz-Rechenzentrum Garching, which was used for a substantial part of the computations.

\section{Appendix A: Benchmark between GENE and GKW}

In Ref. 41, a benchmark between (the local versions of) GENE and GKW based on the same four L-mode cases as in the present work was presented. Complementing the model validation of this paper, it is useful to also verify the correct implementation of the model (particularly its more sophisticated features, e.g. the experimental geometry interface, and the collision operator) for the same cases $^{75}$. Here, we therefore present further details and refinements of the benchmark verification that could not be included in Ref. 41.

The codes GKW ${ }^{40}$ and GENE $^{21}$ have been implemented completely independently, but use equivalent equations and similar numerics, with very similar parallel grids, velocity grids, and collision operators. For this benchmark, both codes were run with the 'nominal' physics setup described in Sec. II, using the full numerical equilibria from the same G-EQDSK files (for GKW, via an interface to the CHEASE code ${ }^{76}$ ), and a linearized Landau-Boltzmann collision operator with both pitch angle and energy scattering including ad-hoc momentum and energy conservation terms. Only deuterium ions and electrons were retained for this benchmark, with $Z_{\text {eff }}$ kept only in the collision frequency. Electromagnetic effects were taken into account, neglecting $\tilde{B}_{\|}$fluctuations.

The linear mode spectra for these settings were shown in Ref. 41 and demonstrated very good agreement between the codes (once the collision operators and geometry inputs were matched exactly). For the nonlinear benchmark, the GENE setup is as described in Secs. II A and IIIC. GKW was run with $60 \times 32 \times 8$ points in the parallel, parallel velocity $\left(v_{\|}\right)$and magnetic moment $(\mu)$ grids respectively (exception: Case $\mathrm{C}$, with only 36 parallel points), and with minimal parallel diffusion for numerical stability and perpendicular spatial diffusion as required to damp the ETG branch. Case A proved to be quite insensitive to grid setups. The other cases were more sensitive, and required perpendicular resolutions to be matched; as is clear from the earlier discussion of Table II, the increased presence of electron driven modes (MTM and TEM) present in cases B, C, and D make them much more challenging for numerical convergence. The grid setups for these cases were matched as closely as practical within stability and resource constraints. The large extent of low- $n$ electron driven modes in balloon- ing space means they are often under-resolved on the nonlinear grid; in these circumstances, differences in the parallel boundary condition between the codes may influence the results. The GKW simulations were run at somewhat larger parallel resolution to remove a numerical instability at low n; subsequently it was found that lower resolutions can be used if an alternative dissipation scheme is used in the parallel differencing.

As discussed in Sec. III C, case D was also run with Dirichlet boundaries (using radial finite-difference versions of both $\operatorname{codes}^{23,77}$ ) to constrain the magnetic streamers. The radial width of the domain at the low field side $\left(L_{r}\right)$ was set to half the shortest perpendicular length scale $0.5 L_{T_{e}}=0.5 \omega_{T_{e}} \mathrm{~d} \rho_{\text {tor }} / \mathrm{d} r=6.3 \mathrm{~cm}$, providing a similar domain size as for standard periodic boundary simulations. Both GENE and GKW used combinations of Krook-type buffer zones at the radial boundaries of the simulation domain, and global sources were applied across the whole radial domain, but with quite different implementations and settings. The electron heat fluxes are relatively robust to these choices, but for a correct ion flux it was important to confirm that the setup prevents profile relaxation and gives a saturated zonal flow. Given the quite different setups, the agreement of the two codes within $20 \%$ in both channels indicates that appropriate setups have been found for both codes for this case. Although the Dirichlet boundaries turned out not to be necessary for this case (see Sec. IIIC), they may still prove useful for local simulations of other edge cases.

The benchmark results for all four cases are summarized in Tab. III and the heat transport spectra are shown in Fig. 13. Note that the simulations shown here exhibit slightly different transport levels from those in Ref. 41, due to extended time averages in the present work, and because of the different collision operator employed in the GKW simulations of cases B and D in Ref. 41. In all cases except $\mathrm{B}$, the agreement between the codes is within the uncertainty of the time average due to intermittency (estimated by taking a sample of time averages). In case $\mathrm{B}$, which is the most difficult for convergence, the codes could not be run with identical grid setups, but still agree within $25 \%$, within the uncertainties of the numerical convergence.

The comparison of the spectra is much more stringent than that of the total flux presented in Ref. 41. The close agreement of the spectral shapes in all cases provides a convincing verification of the nonlinear physics in both codes. To further advance the resolution of the L-mode transport shortfall issue, we have begun to extend this benchmark to additional gyrokinetic codes, whose numerical approaches differ much more substantially than the implementations of GENE and GKW do. Results of this work will be reported in a separate publication. 

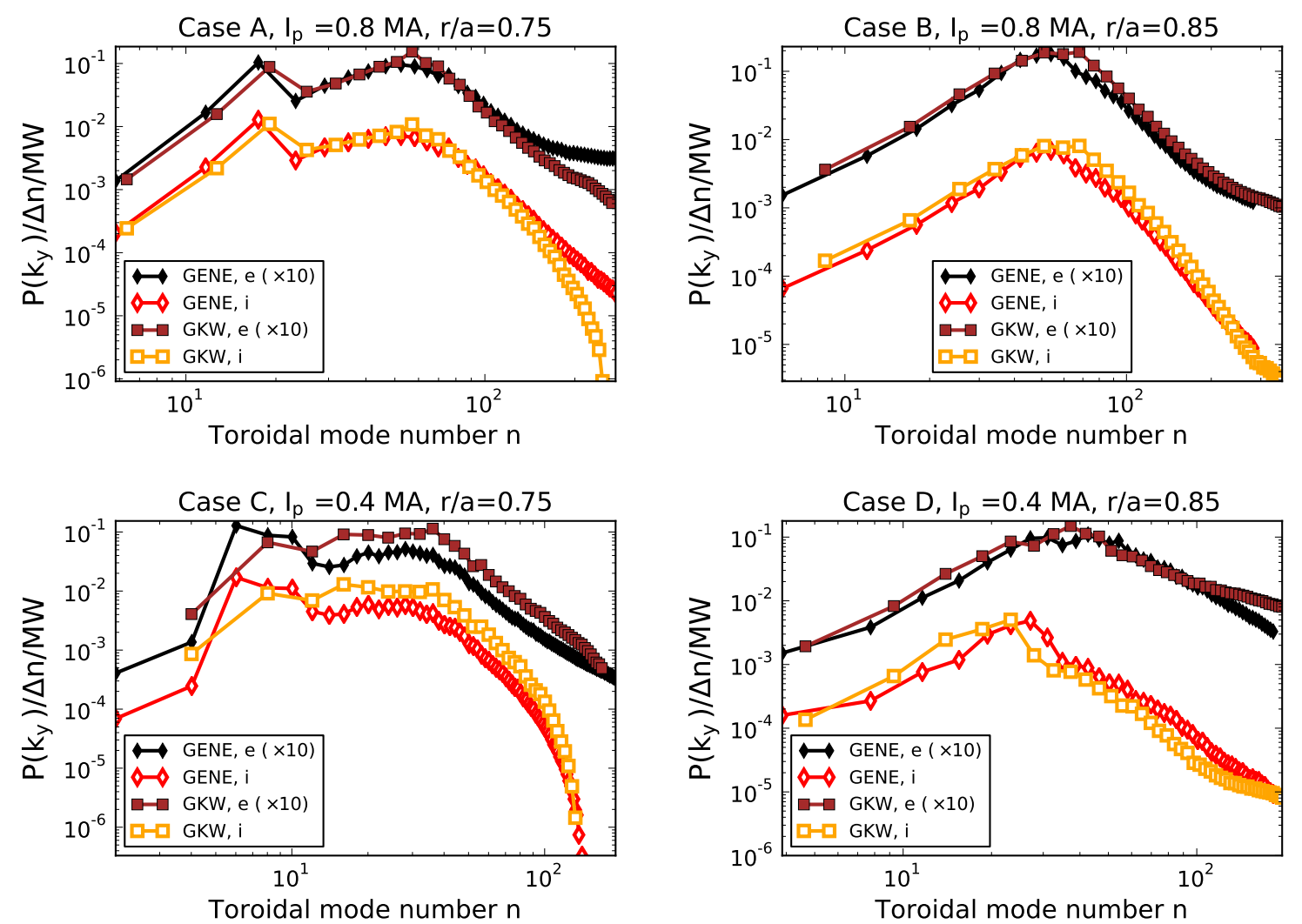

Figure 13: (Color online) Comparison between the GENE and GKW spectra of the electron/ion heat transfer rates for cases A-D.

\begin{tabular}{llllllllll}
$\#$ & Case + comment & $P_{e} / M W$ & $P_{i} / M W$ & $\Gamma T_{i} / Q_{i}$ & $n_{x}$ (ex. buf.) & $n_{y}$ & $\mathcal{N}$ (ex. buf.) & $\Delta n$ & $\Delta t / m s$ \\
\hline \hline 4 & A, GENE & $0.67 \pm 0.11$ & $0.54 \pm 0.14$ & 0.15 & 256 & 48 & 10 & 6 & 1.6 \\
\hline 4B & A, GKW & $0.66 \pm 0.04$ & $0.54 \pm 0.05$ & 0.13 & 255 & 43 & 15 & 6.3 & 0.7 \\
\hline \hline 9 & B, GENE & $0.86 \pm 0.09$ & $0.31 \pm 0.03$ & 0.29 & 256 & 48 & 12 & 6 & 4.3 \\
\hline 9B & B, GKW & $1.11 \pm 0.04$ & $0.45 \pm 0.02$ & 0.28 & 255 & 43 & 18 & 8.5 & 1.4 \\
\hline \hline 11 & C, GENE & $0.47 \pm 0.05$ & $0.52 \pm 0.05$ & 0.05 & 512 & 96 & 7 & 2 & 1.2 \\
\hline 16B & C, GKW & $0.41 \pm 0.06$ & $0.44 \pm 0.07$ & -0.005 & 339 & 43 & 8 & 4 & 0.5 \\
\hline \hline 18 & D, GENE, Dir. & $0.57 \pm 0.08$ & $0.097 \pm 0.014$ & -0.06 & $512(410)$ & 48 & $13.0(10.4)^{*}$ & 4 & 0.6 \\
\hline 18B & D, GKW, Dir. & $0.62 \pm 0.04$ & $0.081 \pm 0.015$ & -0.16 & $768(528)$ & 43 & $24.7(17.0)^{* *}$ & 4.6 & 0.6
\end{tabular}

Table III: Summary of nonlinear runs in the GENE/GKW benchmark, corresponding to the spectra shown in

Fig. 13. The GENE results and numbering are the same as in Tab. II. The time average in the saturated phase is over $\Delta t$. For cases with Dirichlet boundary conditions, numbers in brackets represent the radial domain size excluding the buffer regions (* GENE width $L_{r} / \mathrm{cm}=6.3(5.1) ;{ }^{*}$ GKW width, $L_{r} / \mathrm{cm}=9.1(6.4)$ ).

\section{REFERENCES}

${ }^{1}$ E. J. Doyle, W. A. Houlberg, Y. Kamada, V. Mukhovatov, T. H. Osborne, A. Polevoi, G. Bateman, J. W. Connor, J. G. Cordey, T. Fujita, X. Garbet, T. S. Hahm, L. D. Horton, A. E. Hubbard, F. Imbeaux, F. Jenko, J. E. Kinsey, Y. Kishimoto, J. Li, T. C. Luce, Y. Martin, M. Ossipenko, V. Parail, A. Peeters, T. L. Rhodes, J. E. Rice, C. M. Roach, V. Rozhansky, F. Ryter, G. Saibene, R. Sartori, A. C. C. Sips, J. A. Snipes, M. Sugihara, E. J. Synakowski, H. Takenaga, T. Takizuka, K. Thomsen, M. R.
Wade, H. R. Wilson, ITPA Transport Physics Topical Group, I. Confinement Database, Modelling Topical Group, I. Pedestal, and Edge Topical Group, Nucl. Fusion 47, 18 (2007).

${ }^{2}$ C. Holland, A. E. White, G. R. McKee, M. W. Shafer, J. Candy, R. E. Waltz, L. Schmitz, and G. R. Tynan, Phys. Plasmas 16, 052301 (2009).

${ }^{3}$ A. E. White, W. A. Peebles, T. L. Rhodes, C. H. Holland, G. Wang, L. Schmitz, T. A. Carter, J. C. Hillesheim, E. J. Doyle, L. Zeng, G. R. McKee, G. M. Staebler, R. E. Waltz, J. C. Deboo, C. C. Petty, and K. H. Burrell, Phys. Plasmas 17, 056103 (2010). 
${ }^{4}$ E. Fable, C. Angioni, J. Hobirk, G. Pereverzev, S. Fietz, T. Hein, and ASDEX Upgrade Team, Nucl. Fusion 51, 043006 (2011).

${ }^{5}$ C. Holland, L. Schmitz, T. L. Rhodes, W. A. Peebles, J. C. Hillesheim, G. Wang, L. Zeng, E. J. Doyle, S. P. Smith, R. Prater, K. H. Burrell, J. Candy, R. E. Waltz, J. E. Kinsey, G. M. Staebler, J. C. Deboo, C. C. Petty, G. R. McKee, Z. Yan, and A. E. White, Phys. Plasmas 18, 056113 (2011).

${ }^{6}$ T. L. Rhodes, C. Holland, S. P. Smith, A. E. White, K. H. Burrell, J. Candy, J. C. DeBoo, E. J. Doyle, J. C. Hillesheim, J. E. Kinsey, G. R. McKee, D. Mikkelsen, W. A. Peebles, C. C. Petty, R. Prater, S. Parker, Y. Chen, L. Schmitz, G. M. Staebler, R. E. Waltz, G. Wang, Z. Yan, and L. Zeng, Nucl. Fusion 51, 063022 (2011).

${ }^{7}$ C. Bourdelle, X. Garbet, R. Singh, and L. Schmitz, Plasma Phys. Controlled Fusion 54, 115003 (2012).

${ }^{8}$ A. E. White, N. T. Howard, M. Greenwald, M. L. Reinke, C. Sung, S. Baek, M. Barnes, J. Candy, A. Dominguez, D. Ernst, C. Gao, A. E. Hubbard, J. W. Hughes, Y. Lin, D. Mikkelsen, F. Parra, M. Porkolab, J. E. Rice, J. Walk, S. J. Wukitch, and A. C.-M. Team, Phys. Plasmas 20, 056106 (2013).

${ }^{9}$ N. T. Howard, A. E. White, M. Greenwald, M. L. Reinke, J. Walk, C. Holland, J. Candy, and T. Görler, Phys. Plasmas 20, 032510 (2013)

${ }^{10}$ G. M. Staebler, J. E. Kinsey, and R. E. Waltz, Phys. Plasmas 14, 055909 (2007).

${ }^{11}$ J. E. Kinsey, G. M. Staebler, and R. E. Waltz, Phys. Plasmas 15, 055908 (2008).

${ }^{12}$ J. Candy and R. E. Waltz, J. Comput. Phys. 186, 545 (2003).

${ }^{13}$ Y. Chen and S. E. Parker, J. Comput. Phys. 189, 463 (2003).

${ }^{14} \mathrm{~T}$. Casper, W. Meyer, G. Jackson, T. Luce, A. Hyatt, D. Humphreys, and F. Turco, Nucl. Fusion 51, 013001 (2011).

${ }^{15}$ V. Parail, R. Albanese, R. Ambrosino, J.-F. Artaud, K. Besseghir, M. Cavinato, G. Corrigan, J. Garcia, L. Garzotti, Y. Gribov, F. Imbeaux, F. Koechl, C. Labate, J. Lister, X. Litaudon, A. Loarte, P. Maget, M. Mattei, D. McDonald, E. Nardon, G. Saibene, R. Sartori, and J. Urban, Nucl. Fusion 53, $113002(2013)$.

${ }^{16}$ R. E. Waltz and Z. Deng, Phys. Plasmas 20, 012507 (2013).

${ }^{17}$ B. D. Scott, Phys. Rev. Lett. 65, 3289 (1990).

${ }^{18}$ B. D. Scott, Plasma Phys. Controlled Fusion 45, A385 (2003).

${ }^{19}$ B. D. Scott, Phys. Plasmas 12, 062314 (2005).

${ }^{20}$ A. Kallenbach, J. Adamek, L. Aho-Mantila, S. Äkäslompolo, C. Angioni, C. V. Atanasiu, M. Balden, K. Behler, E. Belonohy, A. Bergmann, M. Bernert, R. Bilato, V. Bobkov, J. Boom, A. Bottino, F. Braun, M. Brüdgam, A. Buhler, A. Burckhart, A. Chankin, I. G. J. Classen, G. D. Conway, D. P. Coster, P. de Marné, R. D'Inca, R. Drube, R. Dux, T. Eich, N. Endstrasser, K. Engelhardt, B. Esposito, E. Fable, H.-U. Fahrbach, L. Fattorini, R. Fischer, A. Flaws, H. Fünfgelder, J. C. Fuchs, K. Gál, M. García Muñoz, B. Geiger, M. Gemisic Adamov, L. Giannone, C. Giroud, T. Görler, S. da Graca, H. Greuner, O. Gruber, A. Gude, S. Günter, G. Haas, A. H. Hakola, D. Hangan, T. Happel, T. Hauff, B. Heinemann, A. Herrmann, N. Hicks, J. Hobirk, H. Höhnle, M. Hölzl, C. Hopf, L. Horton, M. Huart, V. Igochine, C. Ionita, A. Janzer, F. Jenko, C.-P. Käsemann, S. Kálvin, O. Kardaun, M. Kaufmann, A. Kirk, H.-J. Klingshirn, M. Kocan, G. Kocsis, H. Kollotzek, C. Konz, R. Koslowski, K. Krieger, T. Kurki-Suonio, B. Kurzan, K. Lackner, P. T. Lang, P. Lauber, M. Laux, F. Leipold, F. Leuterer, A. Lohs, N. C. Luhmann, Jr., T. Lunt, A. Lyssoivan, H. Maier, C. Maggi, K. Mank, M.-E. Manso, M. Maraschek, P. Martin, M. Mayer, P. J. McCarthy, R. McDermott, H. Meister, L. Menchero, F. Meo, P. Merkel, R. Merkel, V. Mertens, F. Merz, A. Mlynek, F. Monaco, H. W. Müller, M. Münich, H. Murmann, G. Neu, R. Neu, B. Nold, J.-M. Noterdaeme, H. K. Park, G. Pautasso, G. Pereverzev, Y. Podoba, F. Pompon, E. Poli, K. Polochiy, S. Potzel, M. Prechtl, M. J. Püschel, T. Pütterich, S. K. Rathgeber, G. Raupp, M. Reich, B. Reiter, T. Ribeiro, R. Riedl, V. Rohde, J. Roth, M. Rott, F. Ryter, W. Sandmann, J. Santos, K. Sassenberg, P. Sauter, A. Scarabosio, G. Schall, K. Schmid, P. A. Schneider, W. Schnei- der, G. Schramm, R. Schrittwieser, J. Schweinzer, B. Scott, M. Sempf, F. Serra, M. Sertoli, M. Siccinio, A. Sigalov, A. Silva, A. C. C. Sips, F. Sommer, A. Stäbler, J. Stober, B. Streibl, E. Strumberger, K. Sugiyama, W. Suttrop, T. Szepesi, G. Tardini, C. Tichmann, D. Told, W. Treutterer, L. Urso, P. Varela, J. Vincente, N. Vianello, T. Vierle, E. Viezzer, C. Vorpahl, D. Wagner, A. Weller, R. Wenninger, B. Wieland, C. Wigger, M. Willensdorfer, M. Wischmeier, E. Wolfrum, E. Würsching, D. Yadikin, Q. Yu, I. Zammuto, D. Zasche, T. Zehetbauer, Y. Zhang, M. Zilker, and H. Zohm, Nucl. Fusion 51, 094012 (2011).

${ }^{21}$ F. Jenko, W. Dorland, M. Kotschenreuther, and B. N. Rogers, Phys. Plasmas 7, 1904 (2000).

${ }^{22}$ T. Dannert and F. Jenko, Phys. Plasmas 12, 072309 (2005).

${ }^{23}$ T. Görler, X. Lapillonne, S. Brunner, T. Dannert, F. Jenko, F. Merz, and D. Told, J. Comput. Phys. 230, 7053 (2011).

${ }^{24}$ A. Ivanov, R. Khayrutdinov, S. Y. Medvedev, and Y. Y. Poshekhonov, in Proceedings of 32nd EPS Conference on Plasma Phys., Tarragona, 27 June-1 July 2005 ECA, Vol. 29 (2005) pp. 5-063.

${ }^{25}$ P. Xanthopoulos and F. Jenko, Phys. Plasmas 13, 092301 (2006).

${ }^{26}$ P. Xanthopoulos, W. A. Cooper, F. Jenko, Y. Turkin, A. Runov, and J. Geiger, Phys. Plasmas 16, 082303 (2009).

${ }^{27}$ D. Told, F. Jenko, P. Xanthopoulos, L. Horton, E. Wolfrum, and the ASDEX Upgrade Team, Phys. Plasmas 15, 102306 (2008).

${ }^{28}$ B. Scott, Phys. Plasmas 8, 447 (2001).

${ }^{29}$ D. Told and F. Jenko, Phys. Plasmas 17, 042302 (2010).

${ }^{30}$ A. M. Dimits, G. Bateman, M. A. Beer, B. I. Cohen, W. Dorland, G. W. Hammett, C. Kim, J. E. Kinsey, M. Kotschenreuther, A. H. Kritz, L. L. Lao, J. Mandrekas, W. M. Nevins, S. E. Parker, A. J. Redd, D. E. Shumaker, R. Sydora, and J. Weiland, Phys. Plasmas 7, 969 (2000).

${ }^{31}$ W. M. Nevins, J. Candy, S. Cowley, T. Dannert, A. Dimits, W. Dorland, C. Estrada-Mila, G. W. Hammett, F. Jenko, M. J. Pueschel, and D. E. Shumaker, Phys. Plasmas 13, 122306 (2006).

${ }^{32}$ W. M. Nevins, S. E. Parker, Y. Chen, J. Candy, A. Dimits, W. Dorland, G. W. Hammett, and F. Jenko, Phys. Plasmas 14, 084501 (2007).

${ }^{33}$ X. Lapillonne, B. F. McMillan, T. Görler, S. Brunner, T. Dannert, F. Jenko, F. Merz, and L. Villard, Phys. Plasmas 17, 112321 (2010).

${ }^{34}$ B. F. McMillan, X. Lapillonne, S. Brunner, L. Villard, S. Jolliet, A. Bottino, T. Görler, and F. Jenko, Phys. Rev. Lett. 105, 155001 (2010).

${ }^{35}$ J. W. Connor, R. J. Hastie, and J. B. Taylor, Phys. Rev. Lett. 40, 396 (1978)

${ }^{36}$ X. Lapillonne, S. Brunner, T. Dannert, S. Jolliet, A. Marinoni, L. Villard, T. Görler, F. Jenko, and F. Merz, Phys. Plasmas 16, 032308 (2009).

${ }^{37}$ R. L. Miller, M. S. Chu, J. M. Greene, Y. R. Lin-Liu, and R. E. Waltz, Phys. Plasmas 5, 973 (1998).

${ }^{38}$ R. V. Bravenec, J. Candy, M. Barnes, and C. Holland, Phys. Plasmas 18, 122505 (2011).

${ }^{39}$ M. A. Beer, S. C. Cowley, and G. W. Hammett, Phys. Plasmas 2, 2687 (1995)

${ }^{40}$ A. G. Peeters, Y. Camenen, F. J. Casson, W. A. Hornsby, A. P. Snodin, D. Strintzi, and G. Szepesi, Comput. Phys. Commun. 180, 2650 (2009)

${ }^{41}$ E. Fable, C. Angioni, F. J. Casson, D. Told, A. A. Ivanov, F. Jenko, R. M. McDermott, S. Y. Medvedev, G. V. Pereverzev, F. Ryter, W. Treutterer, E. Viezzer, and the ASDEX Upgrade Team, Plasma Phys. Controlled Fusion 55, 124028 (2013). 42 J. Candy, Plasma Phys. Controlled Fusion 51, 105009 (2009).

43 J. Luxon and L. Davis, Fusion Technol.; (United States) 8 (1985).

${ }^{44}$ F. Jenko, T. Dannert, and C. Angioni, Plasma Phys. Controlled Fusion 47, B195 (2005).

${ }^{45}$ F. Merz and F. Jenko, Phys. Rev. Lett. 100, 035005 (2008).

${ }^{46}$ T. Görler and F. Jenko, Phys. Plasmas 15, 102508 (2008).

${ }^{47}$ A. Casati, C. Bourdelle, X. Garbet, F. Imbeaux, J. Candy, F. Clairet, G. Dif-Pradalier, G. Falchetto, T. Gerbaud, V. Grand- 
girard, Ö. D. Gürcan, P. Hennequin, J. Kinsey, M. Ottaviani, R. Sabot, Y. Sarazin, L. Vermare, and R. E. Waltz, Nucl. Fusion 49, 085012 (2009).

${ }^{48}$ R. E. Waltz, A. Casati, and G. M. Staebler, Phys. Plasmas 16, 072303 (2009).

${ }^{49}$ M. Kammerer, F. Merz, and F. Jenko, Phys. Plasmas 15, 052102 (2008).

${ }^{50}$ J. E. Roman, M. Kammerer, F. Merz, and F. Jenko, Parallel Computing 36, 339 (2010), parallel Matrix Algorithms and Applications.

${ }^{51}$ F. Merz, C. Kowitz, E. Romero, J. Roman, and F. Jenko, Comput. Phys. Commun. 183, 922 (2012).

${ }^{52}$ T. Görler and F. Jenko, Phys. Rev. Lett. 100, 185002 (2008).

${ }^{53}$ H. Doerk, F. Jenko, M. J. Pueschel, and D. R. Hatch, Phys. Rev. Lett. 106, 155003 (2011).

${ }^{54}$ W. Guttenfelder, J. Candy, S. M. Kaye, W. M. Nevins, E. Wang, R. E. Bell, G. W. Hammett, B. P. LeBlanc, D. R. Mikkelsen, and H. Yuh, Phys. Rev. Lett. 106, 155004 (2011).

${ }^{55}$ H. Doerk, F. Jenko, T. Görler, D. Told, M. J. Pueschel, and D. R. Hatch, Phys. Plasmas 19, 055907 (2012).

${ }^{56}$ M. J. Pueschel, T. Dannert, and F. Jenko, Comput. Phys. Commun. 181, 1428 (2010).

${ }^{57}$ D. Told, Gyrokinetic Microturbulence in Transport Barriers, Ph.D. thesis, University of Ulm (2012).

${ }^{58}$ A. Arakawa, J. Comput. Phys. 1, 119 (1966).

${ }^{59}$ G. Pereverzev and P. Yushmanov, IPP 5, 42 (1991).

${ }^{60}$ H. Doerk, Gyrokinetic Simulation of Microtearing Turbulence, Ph.D. thesis, University of Ulm (2013).
${ }^{61}$ F. L. Hinton and R. D. Hazeltine, Rev. Mod. Phys. 48, 239 (1976).

${ }^{62}$ B. Scott, A. Kendl, and T. Ribeiro, Contrib. Plasm. Phys. 50, 228 (2010).

${ }^{63}$ This simulation behaves very similarly to those of case D and gives $P_{\mathrm{e}}=0.61 \mathrm{MW}$ and $P_{i}=0.08 \mathrm{MW}$.

${ }^{64}$ M. J. Pueschel and F. Jenko, Phys. Plasmas 17, 062307 (2010).

${ }^{65}$ F. Merz and F. Jenko, Nucl. Fusion 50, 054005 (2010).

${ }^{66}$ N. Winsor, J. L. Johnson, and J. M. Dawson, Phys. Fluids 11, 2448 (1968).

${ }^{67}$ C. Bourdelle, X. Garbet, F. Imbeaux, A. Casati, N. Dubuit, R. Guirlet, and T. Parisot, Phys. Plasmas 14, 112501 (2007).

${ }^{68}$ F. Jenko, W. Dorland, and G. W. Hammett, Phys. Plasmas 8, 4096 (2001).

${ }^{69}$ W. Dorland, F. Jenko, M. Kotschenreuther, and B. N. Rogers, Phys. Rev. Lett. 85, 5579 (2000).

${ }^{70}$ F. Jenko and W. Dorland, Phys. Rev. Lett. 89, 225001 (2002).

${ }^{71}$ F. Jenko, J. Plasma Fusion Res. Ser 6 (2004).

${ }^{72}$ R. E. Waltz, J. Candy, and M. Fahey, Phys. Plasmas 14, 056116 (2007).

${ }^{73}$ M. Barnes, I. G. Abel, W. Dorland, T. GÃ $₫$ rler, G. W. Hammett, and F. Jenko, Phys. Plasmas 17, 056109 (2010).

${ }^{74}$ J. Candy, C. Holland, R. E. Waltz, M. R. Fahey, and E. Belli, Phys. Plasmas 16, 060704 (2009).

${ }^{75}$ M. Greenwald, Phys. Plasmas 17, 058101 (2010).

${ }^{76}$ H. Lütjens, A. Bondeson, and O. Sauter, Comput. Phys. Commun. 97, 219 (1996).

${ }^{77}$ P. Migliano, Y. Camenen, F. J. Casson, W. A. Hornsby, and A. G. Peeters, Phys. Plasmas 20, 022101 (2013). 